\title{
Nonequilibrium transverse motion and emittance growth in ultrarelativistic space-charge dominated beams
}

\author{
S. G. Anderson and J. B. Rosenzweig \\ Department of Physics and Astronomy, UCLA, 405 Hilgard Avenue, Los Angeles, California 90095
}

(Received 3 March 2000; published 14 September 2000)

\begin{abstract}
The transverse dynamics of space-charge dominated beams are investigated both analytically and computationally, in order to understand the mechanisms for emittance oscillations and growth due to nonlinear space-charge fields. This work explores the role of space-charge dominated equilibrium and its relationship to phase space wave breaking, which is responsible for the irreversible emittance growth in these systems. The physics of both coasting and accelerating beams are examined in order to illuminate the most effective approaches to beam handling during the emittance compensation process as well as during subsequent beam transport. These results are discussed within the context of recent ultrahigh brightness rf photoinjector designs.
\end{abstract}

PACS numbers: 05.45.-a, 29.27.Bd, 41.75.Ht, 41.85.Ja

\section{INTRODUCTION}

In recent years, a concerted attempt has been made to understand the space-charge dominated beam dynamics of intense electron beams, mainly in the context of rf photoinjectors. The ultrashort beams in these devices undergo transverse expansion from the photocathode in the initial cell of the rf gun, an expansion accompanied by rapid rms emittance growth [1]. This growth has been found to be due in large part to correlations between the transverse phase space angle described by the rms beam size $\sigma$ and divergence $\sigma^{\prime}$ and the longitudinal position in the beam [2]. A transverse cross section of the beam at a given longitudinal position is referred to as a beam slice, and removal of the correlation between slice position and rms phase space angle $\sigma^{\prime} / \sigma$ through appropriate space-charge dominated beam manipulation is a process known as emittance compensation [2,3]. As discussed in the following section, this process is explainable in terms of linear plasma oscillations (the beam is considered to be a nearly laminar, cold relativistic plasma) about equilibria dictated by the value of the current at a given slice and the applied external forces. This analysis, originally performed by Serafini and Rosenzweig (SR) [4], led to the identification of a new type of space-charge dominated beam equilibrium which is found in accelerating systems, termed the invariant envelope. It was proposed in this analysis that the invariant envelope is the preferred mode of beam propagation for providing optimized emittance compensation. In fact, this point of view is not completely consistent, as we shall see, with the original proposed mechanism of emittance compensation. Part of the motivation for this work is to clarify the role of the invariant envelope in the emittance compensation process.

Further, because the invariant envelope is a generalized equilibrium, a beam slice matched to it in general undergoes wave breaking in the transverse phase space-where the previously approximately single-valued distribution of transverse momenta $f_{p_{x}}(x)$ becomes multiple valuedthus causing an irreversible emittance growth. This emittance growth mechanism has been studied extensively in the field of heavy-ion fusion in the context of Brillouin flow (the rigid rotor equilibrium corresponding to maximum beam density [5]) in coasting, solenoid-focused beams. It is well understood from the viewpoint of microscopic phase space dynamics of coasting beams, as studied by Anderson [6], and alternatively as the conversion of so-called nonlinear field energy to thermal energy, and thus emittance [6-8]. This irreversible emittance growth has also been associated in O'Shea's analysis with the increase in the beam entropy [9]. The fact that initially nonuniform density beams which are matched to external focusing channels undergo wave breaking due to nonlinear space-charge fields is, at first glance, apparently at odds with the assertion that the invariant envelope (a generalized form of matching) is the preferred mode of transport in pulsed, space-charge dominated beams. This work is also intended to address and clarify this apparent disagreement. In the process we also hope to inject some valuable methods from space-charge dominated ion beams $[10,11]$ into the study of emittance compensation of high brightness electron beams. In particular, since the present work is concerned primarily with purely transverse microscopic dynamics associated with beams undergoing reversible and irreversible emittance growth, the connection of our results is most pronounced with Anderson's study of microscopic dynamics in space-charge dominated ion beams. Conversely, in the cases of present interest we are not concerned with long-range irreversible behavior, such as halo formation equipartitioning of energy between phase planes, and so we do not find it necessary to utilize thermodynamics-based tools more familiar to the ion beam community.

Therefore, we can state that this paper is concerned with the self-consistent phase space dynamics of beam slices as they evolve under the influences of space-charge and 
external forces. We analytically study these dynamics to determine the conditions under which phase space wave breaking occurs, for coasting beams, in slab-symmetric, as well as cylindrically symmetric, geometries. The slabsymmetric case is included mainly to allow use of exact and physically transparent results, which illustrate the mechanisms involved in phase space wave breaking. In practice, one is nearly always concerned with cylindrically symmetric beams, and so we extend our discussion of this case to include acceleration in an rf structure. Because the analysis of dynamics of this system is not tractable after wave breaking has occurred, we then also employ computational simulations to further our understanding of the cylindrically symmetric beam physics in both the coasting and accelerating cases. The results of this analysis show that, in order to compensate the beam emittance within a slice, in the presence of significant nonlinearities in the space-charge field, one must avoid matching of the beam to the generalized equilibria (e.g., Brillouin flow, or invariant envelope), and that the optimal transport of a space-charge dominated beam is typically not close to such equilibria.

\section{ENVELOPE DYNAMICS AND LINEAR EMITTANCE COMPENSATION}

The purpose of this section is to provide a review of the analytical theory of emittance compensation as formulated by SR in Ref. [4]. This background is needed in order to understand the detailed nature of the problems addressed in this paper. The invariant envelope theory begins with the writing of the cylindrically symmetric rms envelope equation of each beam slice in the long-beam (twodimensional) limit. This limit is reached when the beam is highly relativistic $\nu_{b} \rightarrow c(\gamma \gg 1)$, and even a short pulse of particles appears elongated in the longitudinal dimension in its rest frame. In this limit, which is assumed for the remainder of this section, the transverse defocusing due to space-charge forces is dependent only on the local value of the current $I(\zeta)=q \lambda(\zeta) \nu_{b} \cong q \lambda(\zeta) c$ and the rms beam size at the particular slice in question $\sigma_{r}(\zeta, z)$, and the envelope equation including acceleration is

$$
\begin{gathered}
\sigma_{r}^{\prime \prime}(\zeta, z)+\left(\frac{\gamma^{\prime}}{\gamma(z)}\right) \\
\sigma_{r}^{\prime}(\zeta, z)+\frac{\eta}{8}\left(\frac{\gamma^{\prime}}{\gamma(z)}\right)^{2} \sigma_{r}(\zeta, z) \\
=\frac{r_{e} \lambda(\zeta)}{\gamma(z)^{3} \sigma_{r}(\zeta, z)} .
\end{gathered}
$$

Here $\zeta=z-c t$ is the internal longitudinal coordinate of a fixed position moving beam (and thus labels a slice), $z$ is the distance along the beam propagation direction, and we have suppressed the thermal emittance term, which means we are assuming a highly space-charge dominated beam. Note that this is a relatively extreme limit, where the thermal motion of particles is completely negligible, and the motion of the ensemble of beam particles resembles that of a cold fluid-a one-component relativistic plasma. In more standard accelerator terminology, this limit corresponds to full depression of the betatron tune by spacecharge defocusing. This type of ultrarelativistic cold-fluid (laminar) beam analysis gives an excellent approximation when one studies high brightness electron beams, but is less applicable in the context of ion beams, which are typically not as extremely space-charge dominated.

Also, the parameter $\eta$ is a measure of the second-order focusing, e.g., nonsynchronous rf wave $[12,13]$ and/or solenoid focusing [4], applied to the beam as it accelerates with normalized, average (over an rf period) spatial rate $\gamma^{\prime}=q\left\langle E_{z}\right\rangle / m_{0} c^{2}$. For a standing wave accelerator $\eta \cong 1$, while for a disk-loaded traveling wave accelerator it is an order of magnitude smaller [13]. If solenoid focusing is also applied, $\eta \rightarrow \eta+2 b^{2}$, where $b=B_{z} /\left\langle E_{z}\right\rangle$. It should be noted that we have adopted the ultrarelativistic limit here mainly to simplify the model of the rf focusing, as well as the mathematics of the analysis. The results we obtain can be straightforwardly generalized to moderately relativistic or even nonrelativistic beams.

When the beam is focused by a solenoid, but not accelerating, $\gamma^{\prime}=0$, we recover the familiar rms envelope equation

$$
\sigma_{r}^{\prime \prime}(\zeta, z)+k_{\beta}^{2} \sigma_{r}(\zeta, z)=\frac{r_{e} \lambda(\zeta)}{\gamma^{3} \sigma_{r}(\zeta, z)},
$$

where $k_{\beta}=q B_{z} / \beta \gamma m_{0} c^{2} \cong q B_{z} / \gamma m_{0} c^{2}$ is the spatial betatron frequency [14], which in this case is identical to the Larmor frequency of the particle. Equation (2) is a nonlinear differential equation with no general analytical solution, but does have a particular equilibrium solution,

$$
\sigma_{\text {eq }}(\zeta)=\frac{1}{k_{\beta}} \sqrt{\frac{r_{e} \lambda(\zeta)}{\gamma^{3}}} .
$$

This steady state envelope given by Eq. (3) corresponds to a rigid rotor equilibrium known as Brillouin flow, in which the beam's canonical angular momentum is 0 . The typical way of dealing with solution of Eq. (2) is to expand it to first order about its equilibrium, in the parameter $\delta \sigma_{r}=\sigma_{r}-\sigma_{\text {eq }} \ll \sigma_{\text {eq }}$, to obtain

$$
\delta \sigma_{r}^{\prime \prime}(\zeta, z)+k_{\beta}^{2} \delta \sigma_{r}(\zeta, z)=-\frac{r_{e} \lambda(\zeta)}{\gamma^{3} \sigma_{\mathrm{eq}}^{2}(\zeta)} \delta \sigma_{r}(\zeta, z),
$$

or

$$
\delta \sigma_{r}^{\prime \prime}(\zeta, z)+2 k_{\beta}^{2} \delta \sigma_{r}(\zeta, z)=0 .
$$

The general solution for small amplitude motion about the equilibrium associated with each beam slice is thus, assuming for simplicity that all slices are initially launched at the same rms size $\sigma_{r}(\zeta, 0)=\sigma_{r 0}$ with no rms angular motion $\sigma_{r}^{\prime}(\zeta, 0)=0$,

$$
\sigma_{r}(\zeta, z)=\sigma_{r 0}+\left[\sigma_{r 0}-\sigma_{\mathrm{eq}}(\zeta)\right] \cos \left(\sqrt{2} k_{\beta} z\right),
$$

with derivative

$$
\sigma_{r}^{\prime}(\zeta, z)=-\sqrt{2} k_{\beta}\left[\sigma_{r 0}-\sigma_{\mathrm{eq}}(\zeta)\right] \sin \left(\sqrt{2} k_{\beta} z\right) .
$$


In this case the $\left(\sigma_{r}, \sigma_{r}^{\prime}\right)$ trace space trajectory of the envelope is simply an ellipse whose origin is offset to $\left(\sigma_{\text {eq }}, 0\right)$. The mismatched envelopes rotate about this offset position with spatial frequency (wave number) $\sqrt{2} k_{\beta}$, which is equal to the plasma frequency $k_{p}=\sqrt{4 \pi r_{c} n_{b} / \beta^{2} \gamma^{3}}$ of the equivalent uniform density $\left[n_{b}(\zeta)=\lambda(\zeta) / 2 \pi \sigma_{r}^{2}\right]$ matched beam [6]. In the small amplitude limit, the oscillation frequency is independent of $\lambda(\zeta)$. Thus every trajectory of this form aligns in trace space twice per plasma period, points at which the projected rms emittance obtained by summing $(\langle\rangle)$ over the ensemble of beam slices in this trace space,

$$
\varepsilon_{r}=\sqrt{\left\langle\sigma_{r}^{2}\right\rangle\left\langle\sigma_{r}^{\prime 2}\right\rangle-\left\langle\sigma_{r} \sigma_{r}^{\prime}\right\rangle^{2}}
$$

vanishes. This definition of emittance is identical to that of the standard radial $\mathrm{rms}$ emittance $\varepsilon_{\mathrm{rms}}=$ $\sqrt{\left\langle r^{2}\right\rangle\left\langle r^{\prime 2}\right\rangle-\left\langle r r^{\prime}\right\rangle^{2}}$ if each slice of the beam is a line in $\left(r, r^{\prime}\right)$ trace space, which connects the origin to the edge of the slice distribution through the value $\left(\sigma_{r}, \sigma_{r}^{\prime}\right)$. This case, which is physically realized when the beam's density distribution is uniform inside of the radius $2=\sqrt{2} \sigma_{r}$ and vanishing outside of this radius, was the subject of the envelope dynamics analyzed in Ref. [4].

In the case most relevant to the emittance compensation process, the beam is launched with a size smaller than equilibrium for all portions of the beam, and the trace space trajectories for various slices are nested ellipses. This is shown in Fig. 1, which displays three elliptical trajectories corresponding to three different slices with $\lambda_{1}<\lambda_{2}<\lambda_{3}$. These ellipses are traversed, according to our linear analysis, with the same frequency. Thus the area in trace space that the points on the three ellipses describe when connected to the trace space origin (at an instant in time), which is proportional to the emittance defined by Eq. (8), oscillates with twice the mismatch oscillation fre-

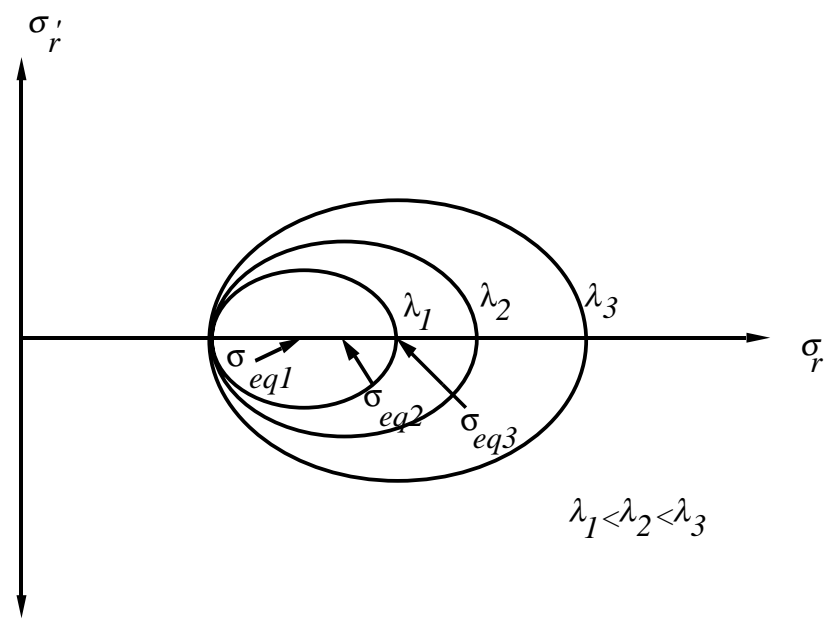

FIG. 1. Trace space trajectories for $\left(\sigma_{r}, \sigma_{r}^{\prime}\right)$ in a system launched with size below the equilibrium for three representative slices, with line charges $\lambda_{1}<\lambda_{2}<\lambda_{3}$. Oscillations proceed at the same frequency $\left(k_{p}=\sqrt{2} k_{\beta}\right)$ about different equilibrium values of $\sigma_{r}$. quency. This phenomenon is illustrated in Fig. 2, which displays the trace space area described by the three slices at $k_{p} z=0, \pi / 2,3 \pi / 2,2 \pi$. It can be seen that the trajectories fan out to produce a large summed (or projected) emittance at $k_{p} z=\pi / 2,3 \pi / 2$, while to lowest order the emittance vanishes at $k_{p} z=0,2 \pi$ and also at $k_{p} z=\pi$ (not shown). These emittance oscillations repeat twice every plasma oscillation, but eventually decohere due to small, higher order differences in the nonlinear plasma frequency in each slice [15]. The proper execution of such an emittance oscillation due to differential slice motion is termed emittance compensation in the context of high current, space-charge dominated beams in rf photoinjectors. This simple picture is complicated somewhat by acceleration, as discussed below, but essentially illustrates the relevant physics of the compensation process.

The picture of the slice dynamics displayed in the trace space diagrams of Figs. 1 and 2 assumes-as is true of motion originating at a cathode in an $\mathrm{rf}$ photoinjector - that the beam expands from its initial size, exceeds an equilibrium value, and finally returns to its initial state. As this is not the most general case, a more complicated, but equally relevant, picture is displayed in Fig. 3, where only two of the slices are launched with sizes below equilibrium, but the third has low enough line charge density that, at the same initial size of the other two slices, it is above equilibrium. This picture displays what happens if a beam is launched with size matched in an rms, integrated beam sense, so that all slices are the same size, but due to variations in current, some slice sizes are initially above, and others below, equilibrium. It can be seen that, while the slice dynamics and associated emittance evolution are in some ways different (the maximum emittance is larger in this case), the overall periodicity of the emittance oscillation is the same. The most important way in which the two situations differ is that in Fig. 1 the rms beam

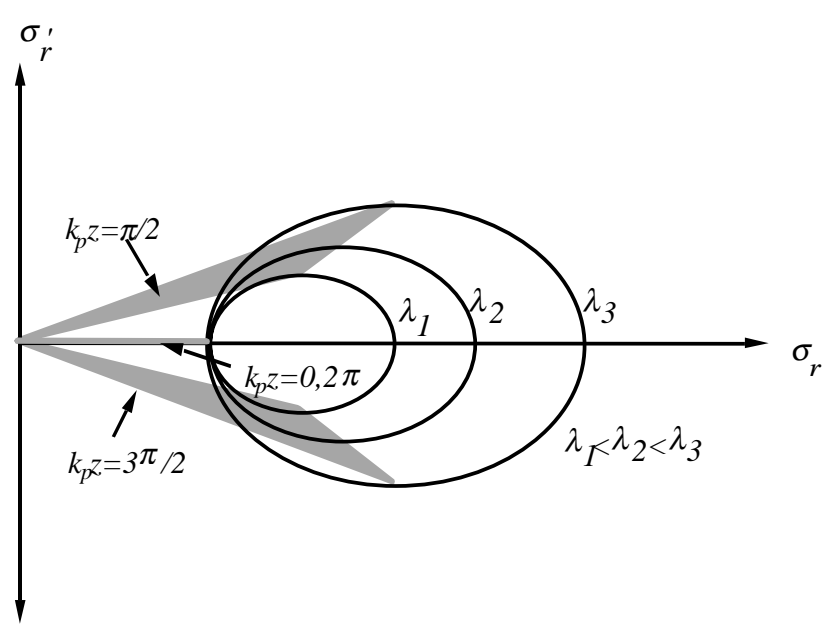

FIG. 2. Projected trace space areas described by the three slices of Fig. 1 , at $k_{p} z=0, \pi / 2,3 \pi / 2,2 \pi$. Note the area (emittance) is maximized at $k_{p} z=\pi / 2,3 \pi / 2$ and vanishes at $k_{p} z=0,2 \pi$ and also at $k_{p} z=\pi$ (not shown). 


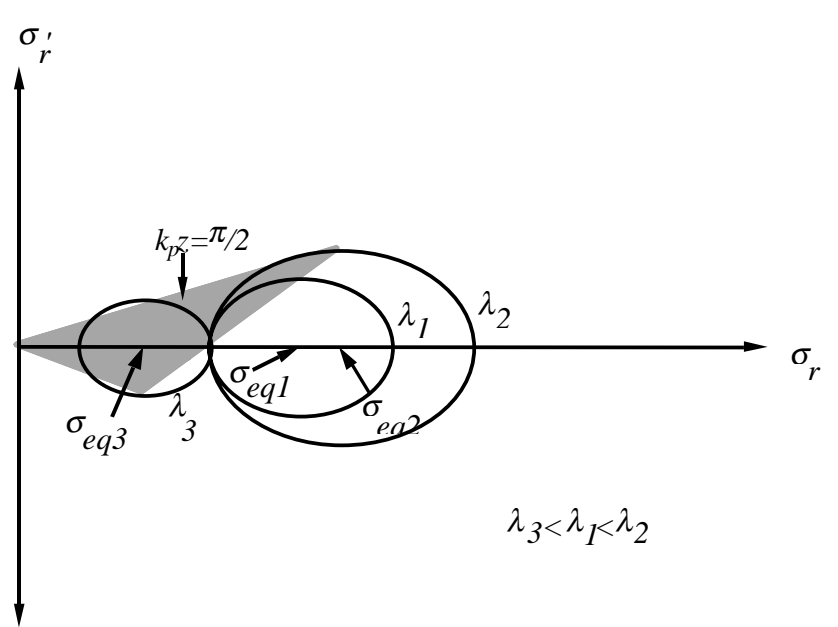

FIG. 3. Projected trace space area described by three slice envelopes with line charge $\lambda_{3}<\lambda_{1}<\lambda_{2}$ with the line charge of slice 3 so low that $\sigma_{r 0}>\sigma_{\text {eq }}$, shown at $k_{p} z=\pi / 2$. The emittance evolution behavior is qualitatively the same as in Figs. 1 and 2, but with larger amplitude of oscillation.

angle $\sigma_{r}^{\prime}$ is the same sign for all slices, while in Fig. 3 the angle of the low current slice is of opposite sign from the other two. We will return to this important point below.

The extension of this type of motion about an equilibrium to a system with longitudinal acceleration has been considered by SR, who have analyzed the motion of such a system with Eq. (1). This equation is again nonlinear, but also has a useful particular solution-which is no longer an equilibrium, however-with which one can begin an analysis, termed the invariant envelope [4],

$$
\sigma_{\text {inv }}(\zeta, z)=\frac{2}{\gamma^{\prime}} \sqrt{\frac{r_{e} \lambda(\zeta)}{(1+\eta / 2) \gamma(z)}} .
$$

It can be seen that the existence of this particular solution is not dependent on external focusing, as even with $\eta=0$ (pure traveling wave, no solenoid) the state corresponding to this solution exists due to the effects of adiabatic damping.

The invariant envelope has the unique property that the trace space angle $\sigma_{r}^{\prime} / \sigma_{r}=-\gamma^{\prime} / 2 \gamma$ is independent of $\lambda(\zeta)$. Thus if one places all slices on their invariant envelope, they will be aligned in trace space angle and the emittance vanishes. It is not possible in practice to do this, and so one must consider what happens when all slices in the beam ensemble are placed close to their invariant envelopes. First, we examine the motion of a slice perturbed slightly off of its invariant envelope, by using a linear expansion of Eq. (1) about this particular solution,

$$
\delta \sigma_{r}^{\prime \prime}+\left(\frac{\gamma^{\prime}}{\gamma}\right) \delta \sigma_{r}^{\prime}+\frac{1+\eta}{4}\left(\frac{\gamma^{\prime}}{\gamma}\right)^{2} \delta \sigma_{r}=0,
$$

where $\delta \sigma_{r}=\sigma_{r}-\sigma_{\text {inv. }}$. This equation has a general form of solution, for the type of initial conditions we have been describing, of

$$
\delta \sigma_{r}=\left[\sigma_{r 0}-\sigma_{\text {inv }}\right] \cos \left[\frac{\sqrt{1+\eta}}{2} \ln \left(\frac{\gamma_{0}}{\gamma(z)}\right)\right],
$$

so that we can write

$$
\sigma_{r}(z)=\sigma_{\mathrm{inv}}+\left[\sigma_{r 0}-\sigma_{\mathrm{inv}}\right] \cos \left[\frac{\sqrt{1+\eta}}{2} \ln \left(\frac{\gamma_{0}}{\gamma(z)}\right)\right],
$$

and

$$
\begin{aligned}
\sigma_{r}^{\prime}(z)= & \frac{\sqrt{1+\eta}}{2} \frac{\gamma^{\prime}}{\gamma(z)}\left[\sigma_{r 0}-\sigma_{\text {inv }}\right] \\
& \times \cos \left[\frac{\sqrt{1+\eta}}{2} \ln \left(\frac{\gamma_{0}}{\gamma(z)}\right)\right],
\end{aligned}
$$

with $\gamma_{0}=\gamma(0)$. Thus the mismatch envelope dynamics are not conceptually much different than in the coasting beam case, with oscillations about the particular solution (no longer an equilibrium, but a secularly diminishing envelope) proceeding at approximately the plasma frequency. Note that the plasma frequency is no longer a constant in this case, but monotonically diminishes with acceleration, approximately as $k_{p} \propto \sqrt{n_{b} / \gamma^{3}} \propto$ $\sigma_{\text {inv }}^{-1} \gamma^{-3 / 2} \propto \gamma^{-1 / 2}$. Mismatch envelope dynamics corresponding to Eqs. (12) and (13) are illustrated by the normalized trace space (phase space) picture given in Fig. 4, which shows the dynamics of three slices corresponding to the hierarchy of currents introduced in Fig. 3.

While the picture in Fig. 4 gives a similar schematic view of emittance oscillations as Fig. 3, it has two notable differences with the nonaccelerating case. The first is simply that the emittance one needs to be concerned with when the beam accelerates is the normalized emittance $\varepsilon_{r, n}=$ $\beta \gamma \sqrt{\left\langle\sigma_{r}^{2}\right\rangle\left\langle\sigma_{r}^{\prime 2}\right\rangle-\left\langle\sigma_{r} \sigma_{r}^{\prime}\right\rangle^{2}} \cong \gamma \sqrt{\left\langle\sigma_{r}^{2}\right\rangle\left\langle\sigma_{r}^{\prime 2}\right\rangle-\left\langle\sigma_{r} \sigma_{r}^{\prime}\right\rangle^{2}}$, which is a measure of the transverse phase space area, and is thus conserved under linear transport and acceleration.

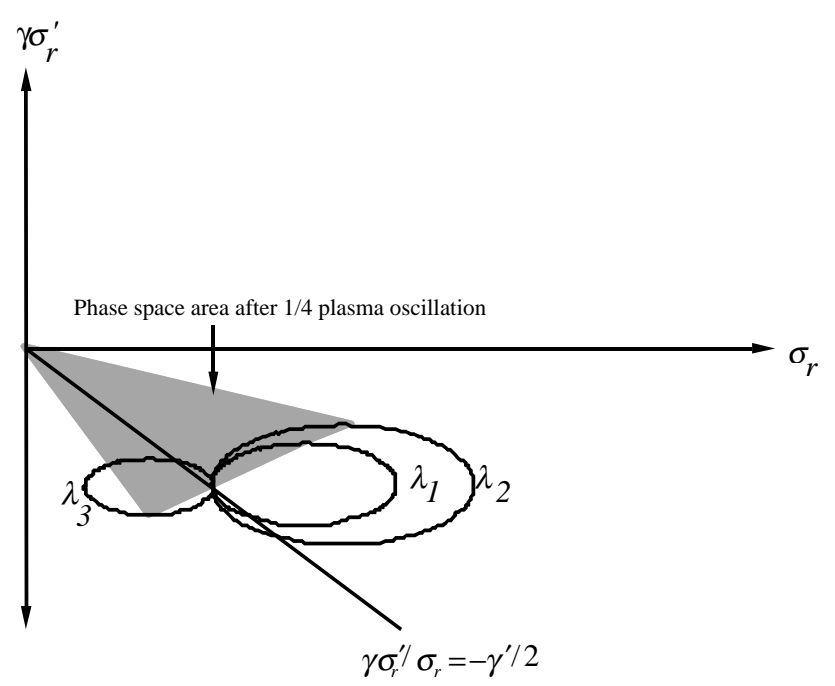

FIG. 4. Normalized, projected trace space areas described by three slices with line charge $\lambda_{3}<\lambda_{1}<\lambda_{2}$ as the envelopes oscillate about the individual invariant envelopes, with the line charge of slice 3 so low that $\sigma_{r 0}>\sigma_{\text {inv }}$. 
The usual "adiabatic damping" of the trace space area, in which the trace space angles diminish through acceleration as $\beta \gamma^{-1}$, is emphasized in Fig. 4 by rescaling of the vertical axis with $\gamma$ (recall that we have set $\beta=1$ in this analysis). This rescaling removes the apparent damping of the motion and makes the diagram approximately a correct phase space plot, in the limit $\beta=1$. The second difference is that all mismatch oscillations have end points attached to a line $\gamma \sigma_{r}^{\prime} / \sigma_{r}=-\gamma^{\prime} / 2$ instead of $\sigma_{r}^{\prime}=0$. As the invariant envelope associated with the slices becomes smaller with increasing energy as $\gamma^{-1 / 2}$ (the ensemble of ellipses shown slides up the line $\gamma \sigma_{r}^{\prime} / \sigma_{r}=-\gamma^{\prime} / 2$ towards the origin), the area associated with the emittance not only oscillates, but secularly damps as $\gamma^{-1 / 2}$.

Note that the offset phase space area described by the mismatch oscillations (the ellipses in Fig. 4) is actually conserved, as can be seen through inspection of Eqs. (12) and (13). This means that for an ensemble of slices placed all at the same initial phase space condition, but with different $\lambda(\zeta)$, the set of points which makes up the section of the phase space boundary not attached to the origin form a line with varying length but no area. This ensemble line stretches and rotates about the invariant envelope of the matched slice. If the invariant envelope slice is actually present in the beam, the ensemble line passes through the invariant envelope line $\gamma \sigma_{r}^{\prime} / \sigma_{r}=-\gamma^{\prime} / 2$, and rotates about the intersection point of these two lines. This intersection is therefore a fixed point in phase space. Thus the matched invariant envelope is a generalized fixed point in the envelope phase space. This is an important observation having implications for particle motion within a slice.

\section{LAMINAR AND NONLAMINAR MOTION IN COASTING SLAB BEAMS}

As can be seen by the analysis above, the self-consistent collective motion of particle beams in cylindrical symmetry is complicated somewhat by the need to approximate the solutions to the relevant differential equations. Because of this, it is most instructive to begin our analysis using a Cartesian, or slab-symmetric (sheet) beam, following the general methods introduced by Anderson in Ref. [6].

We start this discussion by examining a freely expanding (unfocused) laminar beam, with initial $(z=0)$ density profile, infinite in the $y$ and $z$ dimensions, and propagating in the $+z$ direction,

$n_{b}\left(x_{0}\right)=\frac{\Sigma_{b}}{a_{0}} f\left(x_{0}\right)=n_{b 0} f\left(x_{0}\right) \quad$ with $f(0)=1$,

where $\Sigma_{b}$ is the beam charge per unit (slab) area and $a_{0}=$ $\Sigma_{b} / n_{b 0}$ is the effective initial beam width. The case of free expansion can be considered to be the most nonequilibrium scenario possible. It can also be thought of as forming one portion of propagation under periodic application of thin lenses separated by drifts or free-expansion regions.
The equations of motion for the electron position for the free-expansion scenario are, under laminar flow conditions (meaning particle trajectories do not cross),

$$
x^{\prime \prime}(z)=k_{p 0}^{2} F\left(x_{0}\right), \quad F\left(x_{0}\right)=\int_{0}^{x_{0}} f(x) d x=\text { const },
$$

where the local value of the initial (spatial) plasma frequency in the plane of symmetry has been defined as

$$
k_{p 0}^{2}=\frac{4 \pi r_{c} n_{b 0}}{\beta^{2} \gamma^{3}} \text {. }
$$

If laminarity is obeyed, the integral $F\left(x_{0}\right)$ is constant and these equations have solutions dependent only on initial conditions,

$$
x\left(x_{0}, z\right)=x_{0}+\frac{\left(k_{p 0} z\right)^{2}}{2} F\left(x_{0}\right) .
$$

The density distribution is also a simple function of its initial state, as conservation of probability gives $f\left(x\left(x_{0}\right), z\right) d x=f\left(x_{0}\right) d x_{0}$ or

$$
f\left(x\left(x_{0}\right), z\right)=\frac{f\left(x_{0}\right)}{\frac{d x\left(x_{0}\right)}{d x_{0}}}=\frac{f\left(x_{0}\right)}{1+\frac{\left(k_{p 0} z\right)^{2}}{2} f\left(x_{0}\right)} .
$$

In the freely expanding case, the density distribution becomes more uniform as it expands over many plasma radians $\left(k_{p} z \gg 1\right)$,

$$
f\left(x\left(x_{0}\right)\right)=\frac{f\left(x_{0}\right)}{1+\frac{\left(k_{p 0} z\right)^{2}}{2} f\left(x_{0}\right)} \Rightarrow \frac{2}{\left(k_{p 0} z\right)^{2}} .
$$

This observation is critical, as it implies that the transport is "more linear," since the space-charge defocusing for a uniform beam becomes approximately linearly dependent on offset,

$$
x^{\prime \prime}(z)=k_{p 0}^{2} F\left(x_{0}\right) \approx x / 2 z^{2} .
$$

This will in turn imply that the phase space wave-breaking effects which lead to irreversible emittance growth are mitigated, since the angle that a particle attains becomes more linearly correlated with position,

$$
\frac{x^{\prime}}{x}=\frac{k_{p 0}^{2} z F\left(x_{0}\right)}{x_{0}+\frac{1}{2} k_{p 0}^{2} z^{2} F\left(x_{0}\right)} \rightarrow \frac{2}{z},
$$

If the phase space distribution lies along a straight line, the emittance vanishes, so Eq. (21) indicates a desirable trend.

An example of this increased distribution uniformity is shown in Fig. 5, where a beam with initial parabolic profile

$$
f\left(x_{0}\right)=1-\left(\frac{x}{a}\right)^{2}
$$

has freely expanded for a distance $k_{p 0} z=4$. The profile has become noticeably flattened during this expansion.

It is instructive at this point to calculate the emittance evolution associated with the freely expanding beam. In order to do so, we consider a number of possible forms of the distribution: Gaussian, parabolic, and uniform 


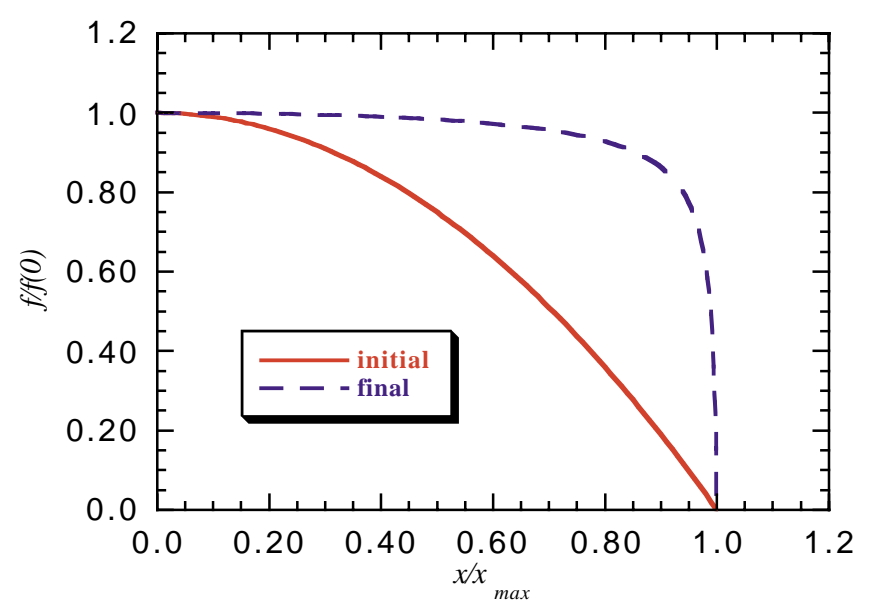

FIG. 5. (Color) Initially parabolic slab beam distribution (solid line) mapped to more uniform (normalized) distribution (dashed line) after a drift length $k_{p 0} z=4$. Distribution shown as a function of relative offset position $x / x_{\max }$.

("flattop"). The single particle equations of motion and the condition of laminar flow allow the calculation of the second moments of the distribution and consequently the rms emittance. Laminar flow implies

$$
n_{b}(x, z) d x=n_{b}\left(x_{0}\right) d x_{0} .
$$

Thus, the second moments of the distribution in trace space can be simply calculated by integrating with respect to the initial particle positions. For example, $\sigma^{2}$ is written as follows:

$$
\left\langle x^{2}\right\rangle=\int_{-\infty}^{\infty} x^{2}\left(x_{0}, z\right) n_{b}\left(x_{0}\right) d x_{0} .
$$

Through this method the second moments are straightforwardly calculated, and the emittance is found. The emittance evolution of the drifting laminar beam can be written in the following general form:

$$
\varepsilon=\alpha k_{p 0}^{2} \sigma_{0} z
$$

where $\sigma_{0}$ is the initial rms spread in the distribution and $\alpha$ is a form factor dependent on the initial beam distribution type. The values of $\alpha$ are summarized in Table I. For a uniform initial distribution, there are no nonlinear forces, and thus no emittance growth. Note that in the case of free expansion the emittance grows linearly with distance

TABLE I. Values of the form factor $\alpha$ for various initial slabsymmetric distribution types.

\begin{tabular}{cc}
\hline \hline Profile & $\alpha$ \\
\hline Gaussian & $\sqrt{(\pi-3) / 3 \pi}$ \\
Parabolic & $\sqrt{2 / 3675}$ \\
Flattop & 0 \\
\hline \hline
\end{tabular}

from the launching point, but has no dependence on initial beam size, as $k_{p 0}^{2} \sigma_{0} \propto \Sigma_{b}$. While this linear growth is a worrisome phenomenon, it turns out not to be valid for cylindrically symmetric beams. In cylindrical beams, the emittance growth is reversed after a time (see the simulation in Fig. 9 below) during expansion, and after application of a thin lens, a nearly perfect oscillation of this nonlinear space-charge force-induced emittance can be made to occur. This compensation of the nonlinearity-derived emittance, which is the central phenomenon under study in this paper, will be discussed in following sections.

Wave breaking occurs in phase space when the value of $x(z)$ somewhere in the distribution becomes independent of $x_{0}$, and the transverse momentum distribution becomes a multiple valued function of transverse offset. According to Eq. (15), this condition $\left(d x / d x_{0}=0\right)$ also implies that the density would become singular at these points. Note that there is no wave breaking for the free-expansion slabsymmetric case, as

$$
\frac{d x}{d x_{0}}=1+\frac{\left(k_{p 0} z\right)^{2}}{2} f\left(x_{0}\right)>1>0 .
$$

This will change when we introduce focusing, but one conclusion remains from this analysis: one must allow the beam to stay far from equilibrium in order to avoid the most serious consequences of wave breaking.

There are two ways to proceed from this point. One is to introduce thin lenses to produce a periodic transport system with an rms matched (in the sense that the envelope has the same periodicity and symmetry as the applied focusing forces) beam. The other is to introduce a uniform strength focusing channel (akin to the solenoid commonly used in cylindrically symmetric systems), but to allow a mismatch between the beam and the channel. In the interest of simplicity, we will follow the latter course first.

In a system with uniform strength focusing, Eq. (15) becomes

$$
x^{\prime \prime}(z)+k_{\beta}^{2} x(z)=k_{p 0}^{2} F\left(x_{0}\right),
$$

where we have introduced the betatron wave number $k_{\beta}$ associated with free oscillations under the influence of the focusing gradient. The equilibrium solution for a given initial particle position is simply

$$
x_{\mathrm{eq}}\left(x_{0}\right)=\frac{k_{p 0}^{2}}{k_{\beta}^{2}} F\left(x_{0}\right) .
$$

This equilibrium can be made consistent for all particles, in the sense that no particles will move after the distribution is launched, if $F\left(x_{0}\right)=1$ and $k_{p 0}^{2}=k_{\beta}^{2}$. If any initial distribution other than a uniform one is employed, there will be subsequent motion and associated rearrangement of the distribution. In this more general case, we may write the solution to Eq. (27) as

$$
x\left(x_{0}, z\right)=x_{\mathrm{eq}}\left(x_{0}\right)+\left[x_{0}-x_{\mathrm{eq}}\left(x_{0}\right)\right] \cos \left(k_{\beta} z\right) .
$$


The wave breaking condition associated with this motion is

$$
\frac{\partial x}{\partial x_{0}}=\frac{k_{p 0}^{2}}{k_{\beta}^{2}} f\left(x_{0}\right)+\left[1-\frac{k_{p 0}^{2}}{k_{\beta}^{2}} f\left(x_{0}\right)\right] \cos \left(k_{\beta} z\right)=0,
$$

or

$$
f\left(x_{0}\right)=-\frac{k_{\beta}^{2}}{2 k_{p 0}^{2}} \frac{\cos \left(k_{\beta} z\right)}{\sin ^{2}\left(k_{\beta} z / 2\right)} .
$$

It can be seen that wave breaking always occurs for a sufficiently small value of $f\left(x_{0}\right)$, i.e., portions of the beam found in a long continuous tail, assuming a monotonically decreasing function $f\left(x_{0}\right)$. Quantitatively, Eq. (31) states that wave breaking eventually occurs for all $f\left(x_{0}\right)<k_{\beta}^{2} / 2 k_{p 0}^{2}$, with the most interior value of $x_{0}$ undergoing wave breaking at $k_{\beta} z=\pi$ (for distributions which smoothly approach zero, wave breaking begins in these tails at $k_{\beta} z=\pi / 2$ ). It is also apparent that wave breaking can be avoided by a combination of removal of the distribution tails, so that $f\left(x_{0}\right)$ discontinuously goes to zero at a hard-edge beam boundary, and by making the ratio $k_{\beta}^{2} / k_{p 0}^{2}$ become small. When this ratio is near unity, the beam is closely "matched" to the external focusing, and when the ratio is much smaller than unity the beam is mismatched, with the focusing being too weak to control the beam distribution at its launch size. An alternative way of understanding wave breaking is to note that the equilibrium beam size $x_{\mathrm{eq}}$ associated with the initial wave-breaking position is a fixed point of the oscillation. On the other hand, we know that the origin in trace space is also a fixed point, with an opposing sense of phase space rotation about it. The existence of two such fixed points guarantees that the trace space will filament after wave breaking and the emittance will grow irreversibly. The trace space picture of this system is displayed in Fig. 6.

Thus we deduce that a mismatched beam is more likely to preserve its laminar flow, under mismatched conditions, which is an extension and deepening of what we have learned from the case of free expansion. To emphasize this point, in Fig. 7 we show a plot of normalized beam density at the maximal wave-breaking point $k_{\beta} z=\pi$ for a cutoff (at the $25 \%$ intensity level) parabolic distribution in nearly matched $\left(k_{\beta}^{2} / k_{p 0}^{2}=4 / 3\right)$ and highly mismatched $\left(k_{\beta}^{2} / k_{p 0}^{2}=1 / 3\right)$ cases. The nearly matched case barely evades wave breaking, and displays a very large density spike at the beam edge, while the highly mismatched beam easily maintains laminarity, giving a much smaller density spike.

In order to calculate the emittance evolution in the case of the slab beam in a focusing channel, we follow the same procedure as in the drifting beam case up to the point of wave breaking, where laminar flow strictly ends

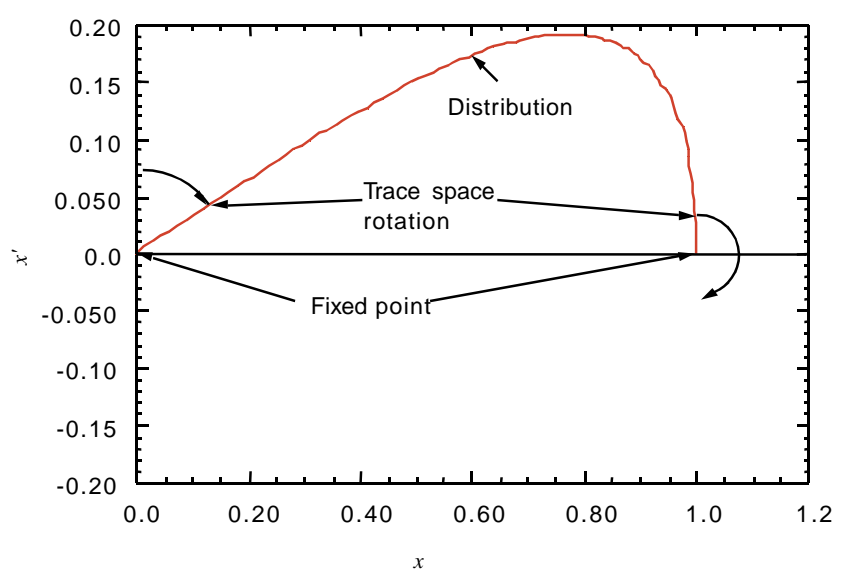

FIG. 6. (Color) Trace space picture of slab symmetric beam at wave breaking onset $\left(k_{\beta} z=\pi / 2\right)$, for the case of $k_{\beta}^{2} / k_{p 0}^{2}=$ $2 / 3$, showing two fixed points with opposing direction of rotation.

and our present analysis breaks down. Assuming a cold beam initially at a waist $\left(x_{0}^{\prime}=0\right)$ the emittance evolution is found to be

$$
\varepsilon=\alpha \frac{k_{p 0}^{2}}{k_{\beta}} \sigma_{0}\left|\sin \left(k_{\beta} z\right)\right|
$$

where, again, $\alpha$ is a constant depending on the form of the initial distribution and the factor $k_{p 0}^{2} \sigma_{0} \propto \Sigma_{b}$ has no dependence on initial beam size. We note from this that the predicted maximum emittance occurs at $k_{\beta} z=\pi / 2$, as with the correlated interslice emittance studied in Ref. [4]. It should also be emphasized that this is the same longitudinal position that the initial wave breaking occurs in for a distribution with a continuous tail.

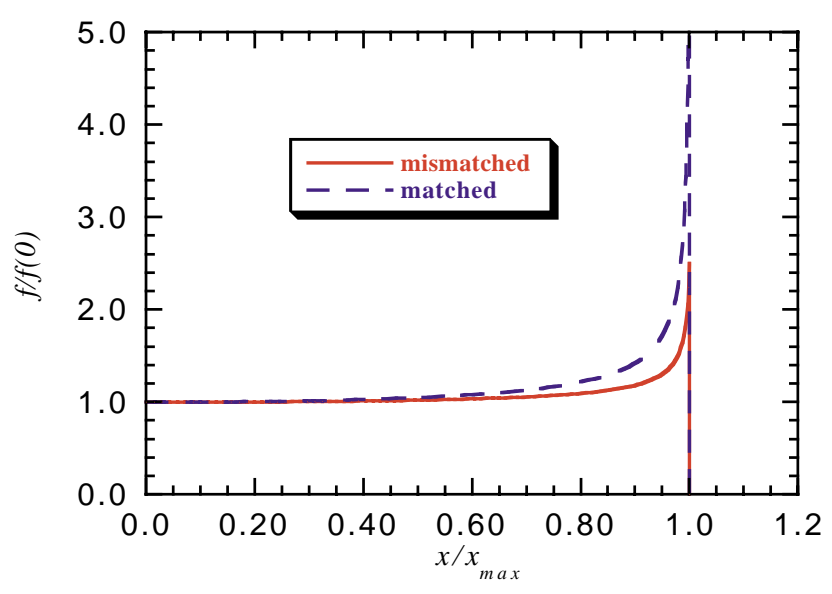

FIG. 7. (Color) Normalized beam density $f / f(0)$ for a beam with initially parabolic slab beam distribution (cutoff at 0.25 normalized density) at $k_{\beta} z=\pi$ for distribution in nearly matched $\left(k_{\beta}^{2} / k_{p 0}^{2}=4 / 3\right)$ and highly mismatched $\left(k_{\beta}^{2} / k_{p 0}^{2}=1 / 3\right)$ cases. Offset $x$ is normalized to its maximum value in the distribution. 


\section{LAMINAR AND NONLAMINAR MOTION IN COASTING CYLINDRICAL BEAMS}

The density of a continuous beam in an axisymmetric system can be described by the expression

$$
n_{b}(r, z)=\lambda_{b} f(r, z),
$$

where $\lambda_{b}=I / q \nu$ is the beam's axial charge density. The electromagnetic force on a particle in such a distribution is

$F_{r}(r, z)=\frac{2 q}{\gamma^{2} r} \int_{0}^{r_{0}} 2 \pi n_{b}(\tilde{r}, z) \tilde{r} d \tilde{r} \equiv \frac{2 q \lambda\left(r_{0}\right)}{\gamma^{2} r}$.

The force has been written in terms of the enclosed current at an initial point $r_{0}\left(z_{0}\right)$,

$$
\lambda\left(r_{0}\right)=\int_{0}^{r_{0}} 2 \pi n_{b}\left(\tilde{r}, z_{0}\right) \tilde{r} d \tilde{r}
$$

which for laminar flow is a constant of the motion.

The equation of motion for a particle with no canonical angular momentum experiencing both a solenoidal restoring force and the repulsive space charge force corresponding to Eq. (34) is

$$
r^{\prime \prime}(z)+k_{\beta}^{2} r(z)=\frac{2 r_{e} \lambda\left(r_{0}\right)}{\beta^{2} \gamma^{3} r} .
$$

Equation (36), like Eq. (2), is a nonlinear equation not amenable to exact solution in general. We can begin an approximate analysis, however, by defining an equilibrium radius corresponding to each value of $r_{0}$,

$$
r_{\mathrm{eq}}\left(r_{0}\right)=\sqrt{\frac{2 r_{e} \lambda\left(r_{0}\right)}{k_{\beta}^{2} \beta^{2} \gamma^{3}}} \equiv r_{0} \frac{\bar{k}_{p}\left(r_{0}\right)}{\sqrt{2} k_{\beta}} .
$$

Here we have introduced an average beam plasma frequency

$$
\bar{k}_{p}^{2}\left(r_{0}\right)=\frac{4 \pi r_{c} \bar{n}_{b}\left(r_{0}\right)}{\beta^{2} \gamma^{3}}=\frac{2 r_{c} \lambda\left(r_{0}\right)}{r_{0}^{2} \beta^{2} \gamma^{3}},
$$

which corresponds to the mean enclosed initial density at $r_{0}$.

We now proceed to linearize Eq. (36) about the equilibria given in Eq. (37) to obtain

$$
\delta r^{\prime \prime}+2 k_{\beta}^{2} \delta r=0,
$$

where $\delta r=r-r_{\mathrm{eq}}$. This equation yields a familiar form of solution, for a distribution beginning with no radial momentum (or angular momentum in the beam's Larmor frame)

$$
r\left(r_{0}, z\right)=r_{\mathrm{eq}}\left(r_{0}\right)+\left[r_{0}-r_{\mathrm{eq}}\left(r_{0}\right)\right] \cos \left(\sqrt{2} k_{\beta} z\right) .
$$

The wave-breaking condition is again given by

$$
\begin{aligned}
\frac{\partial r}{\partial r_{0}} & =\frac{\partial r_{\mathrm{eq}}}{\partial r_{0}}+\left[1-\frac{\partial r_{\mathrm{eq}}}{\partial r_{0}}\right] \cos \left(\sqrt{2} k_{\beta} z\right)=0, \\
\text { or } \frac{\partial r_{\mathrm{eq}}}{\partial r_{0}} & =-\frac{\cos \left(\sqrt{2} k_{\beta} z\right)}{2 \sin ^{2}\left(k_{\beta} z / \sqrt{2}\right)} .
\end{aligned}
$$

The quantity on the left-hand side of Eq. (41) can be written as

$$
\frac{\partial r_{\mathrm{eq}}}{\partial r_{0}}=\frac{r_{p}}{2 \lambda\left(r_{0}\right)} \frac{\partial \lambda}{\partial r_{0}}=\frac{k_{p}^{2}\left(r_{0}\right)}{\sqrt{2} \bar{k}_{p}\left(r_{0}\right)},
$$

where we have employed the local measure of the initial beam plasma frequency,

$$
k_{p}^{2}\left(r_{0}\right)=\frac{4 \pi r_{c} n_{b}\left(r_{0}\right)}{\beta^{2} \gamma^{3}}=\frac{2 r_{c} \lambda\left(r_{0}\right)}{\beta^{2} \gamma^{3} r_{0}^{2}} .
$$

As an illustrative example, let us examine the wavebreaking condition for the case of an initially Gaussian beam, where

$$
n_{b}\left(r_{0}\right)=n_{b 0} \exp \left(-r_{0}^{2} / 2 \sigma_{r}^{2}\right) .
$$

In this case,

$$
\begin{aligned}
\lambda\left(r_{0}\right) & =2 \pi n_{b 0} \int_{0}^{r_{0}} r \exp \left(-r^{2} / 2 \sigma_{r}^{2}\right) d r \\
& =2 \pi n_{b 0} \sigma_{r}^{2}\left[1-\exp \left(-r_{0}^{2} / 2 \sigma_{r}^{2}\right)\right] \\
& =2 \pi \sigma_{r}^{2}\left[n_{b 0}-n_{b}\left(r_{0}\right)\right]
\end{aligned}
$$

and the wave-breaking condition can be written as

$$
\begin{aligned}
\frac{k_{p 0} 0}{k_{\beta}} \frac{r_{0}}{\sigma_{r}} \frac{\exp \left(-r_{0}^{2} / 2 \sigma_{r}^{2}\right)}{\sqrt{1-\exp \left(-r_{0}^{2} / 2 \sigma_{r}^{2}\right)}} & =-\frac{\cos \left(\sqrt{2} k_{\beta} z\right)}{\sin ^{2}\left(k_{\beta} z / \sqrt{2}\right)}, \\
\text { with } k_{p 0}^{2}=k_{p}^{2}(0) & =\frac{4 \pi r_{c} n_{b 0}}{\beta^{2} \gamma^{3}} .
\end{aligned}
$$

For wave breaking to be avoided, we have that the lefthand side of Eq. (46) must be greater than unity,

$$
\frac{k_{p 0}}{k_{\beta}} \frac{r_{0}}{\sigma_{r}} \frac{\exp \left(-r_{0}^{2} / 2 \sigma_{r}^{2}\right)}{\sqrt{1-\exp \left(-r_{0}^{2} / 2 \sigma_{r}^{2}\right)}} \equiv \frac{k_{p 0}}{k_{\beta}} g\left(r_{0}\right)>1 \text {. }
$$

The function $g\left(r_{0}\right)$ is shown in Fig. 8 with $f\left(r_{0}\right)$ also displayed for comparison. It can be seen that $g\left(r_{0}\right)$ approximately follows the density, and thus the threshold for wave breaking is estimated as

$$
\frac{k_{p 0}}{k_{\beta}} f\left(r_{0}\right) \cong 1 .
$$

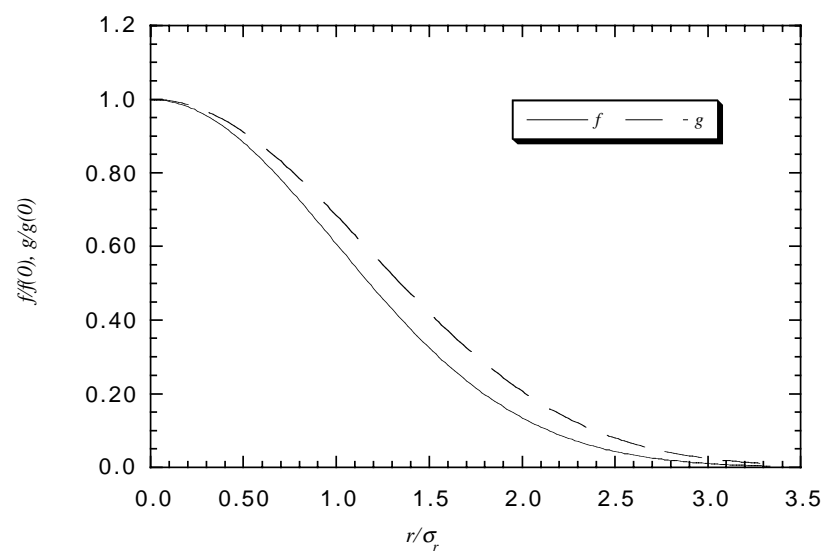

FIG. 8. A comparison of the function $g\left(r_{0}\right)$ with Gaussian $f\left(r_{0}\right)$. 
TABLE II. Values of the form factor $\alpha$ for various initial cylindrical beam slice distribution types.

\begin{tabular}{ll}
\hline \hline Profile & \multicolumn{1}{c}{$\alpha$} \\
\hline Gaussian & 0.141 \\
Parabolic & 0.065 \\
Flattop & 0 \\
\hline \hline
\end{tabular}

This is in contrast to the equivalent condition found in the slab beam case,

$$
\frac{k_{p 0}^{2}}{2 k_{\beta}^{2}} f\left(y_{0}\right) \cong 1,
$$

which has the stronger quadratic dependence on the mismatch parameter $k_{p 0} / k_{\beta}$.

As the linear dynamics of the axisymmetric beam have been seen to be formally quite similar to those of the slab beam, it is not surprising that the emittance evolution is similar as well. Given the same initial conditions as assumed in the slab case, we find the emittance to be of the same form as well,

$$
\varepsilon=\alpha \sigma_{0}^{2} k_{p 0}\left|\sin \left(\sqrt{2} k_{\beta} z\right)\right| .
$$

Here $\alpha$ is again a form factor, defined as in the previous section. The numerical values of $\alpha$ found for the cylindrically symmetric case are shown in Table II. We will see that Eq. (50) provides a very accurate description of the emittance evolution up until wave breaking. Note that the emittance in Eq. (50) is in fact linearly dependent on $\sigma_{0}$, as $k_{p 0} \propto \sigma_{0}^{-1}$.

\section{SIMULATION OF COASTING CYLINDRICAL BEAMS}

The analytical treatments of intraslice transverse space charge detailed above are limited to the laminar flow regime and, in the case of cylindrical beams, are only approximate. They do, however, predict where wave breaking will occur, and that it can be minimized or avoided by mismatching the beam-focusing channel system. In order to test these predictions and examine the behavior of a beam slice after wave breaking, we use self-consistent simulations, using a one-dimensional time dependent code called NORSE, that follow the evolution of the beam using the space-charge force of Eq. (34). To simulate the evolution of a beam slice, the space-charge force of Eq. (34) is calculated by integrating over the beam density at every time step. In order to counter numerical noise inherent in calculating the beam density, we employ the use of Riccian particles, as was introduced in beam simulations in the ITACA code [16]. In this case each particle has a finite size and its charge distribution is given by $\rho_{\text {Ric }}\left(r, r_{i}\right)=q_{i} \exp \left[-\left(r_{i}^{2}+r^{2}\right) / 2 \sigma_{i}^{2}\right] I_{0}\left(r r_{i}\right) / 2 \pi \sigma_{i}^{2}$. With this type of particle distribution the beam density is given simply by the sum of the density contributions of each particle at a given point, $\rho_{b}(r)=\sum_{i} \rho_{\mathrm{Ric}}\left(r, r_{i}\right)$, and the calculation of the space-charge force is straightforward. Because the use of Riccian particles provides very smooth beam densities, it is not feasible to simulate a perfectly hard-edged distribution. In the context of this study, that drawback is not very important since we are interested in studying distributions that will strongly wave break.

We found in the case of the slab beam expanding under its space-charge force that there was no wave breaking for any type of distribution. Equation (34) tells us that this is not the case for a freely expanding cylindrical beam if the initial distribution function falls off, so that the integral of the charge density does not increase proportionally with $r$. In this case we expect wave breaking and look to simulations for understanding of the beam behavior after wave breaking occurs.

The emittance evolution of the freely expanding beam shows the effects of wave breaking. As in the focusing channel, the emittance increases to a maximum at $\lambda_{p 0} / 4$ where wave breaking occurs. While the beam continues to expand, the particles in the vicinity of the initial wave-breaking point (where the maximum outward force is found) effectively rotate about this outward-moving point. This rotation causes the tail particles to "tuck under" in phase space in a distance a bit longer than the initial plasma half-wavelength (the plasma frequency is not constant, but decreases as the beam expands), as would be expected, and the emittance decreases during this initial rotation. The emittance growth is not perfectly compensated by this nonlinear effect, however, and the emittance reaches a local minimum. After that, $\varepsilon$ becomes simply proportional to $\sigma$ as the beam continues to expand. Examination of the beam phase space evolution, shown in Figs. 9 and 10, illustrate this process.

We note from Fig. 10(b) that this tuck under effect on the emittance occurs only after the rms beam size has grown substantially (recall that $k_{p 0} z>\pi$, and the beam has had a large distance in which to expand), as the emittance minimum occurs when $\sigma / \sigma_{0} \approx 8.5$.

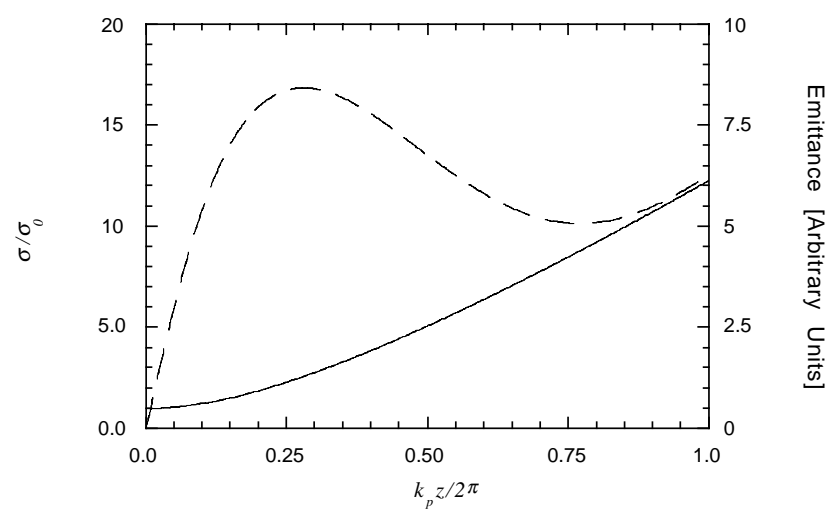

FIG. 9. Results of a simulation of the free-expansion of an initially Gaussian beam. The beam size (solid line) increases monotonically while the emittance (dashed line) has a local maximum and minimum. 


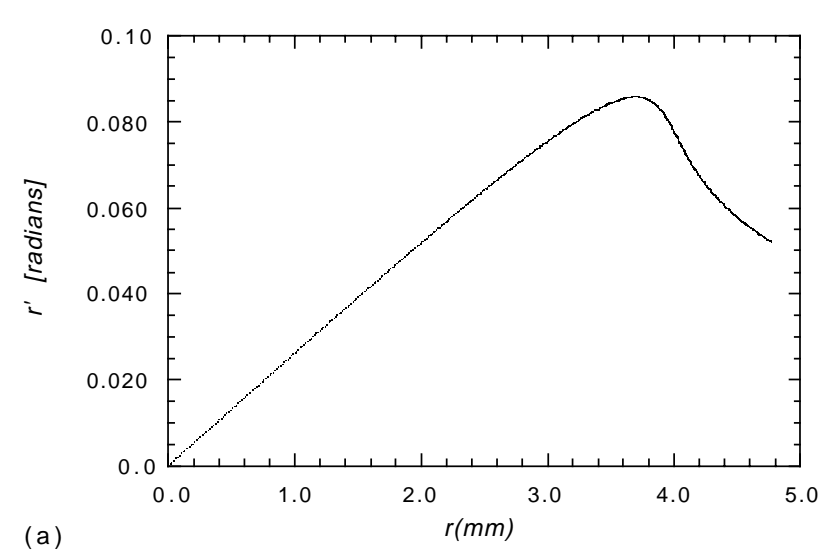

(a)

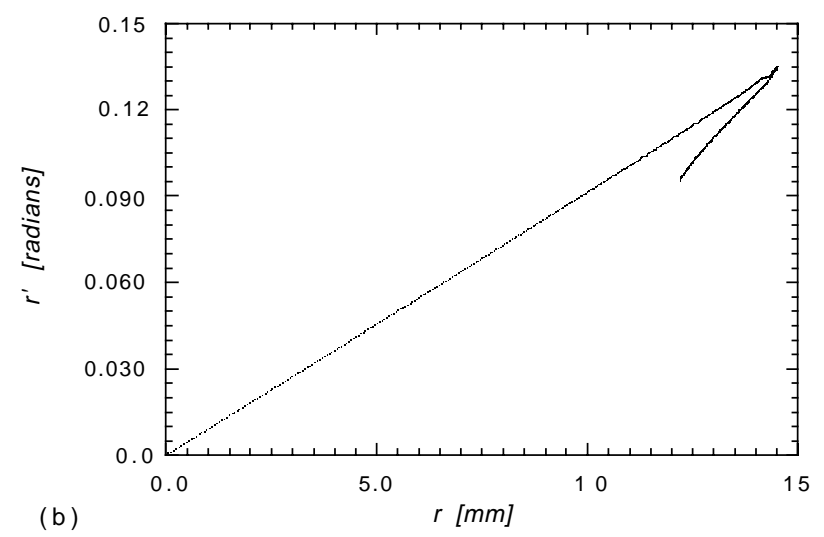

FIG. 10. Trace space plots of a freely expanding, initially Gaussian beam at the initial emittance (a) maximum and (b) minimum.

While the drifting beam is instructive, we are interested in beam transport involving focusing elements. We proceed again as before by examining two cases: periodic thin lenses separated by drifts and a focusing channel. In the case of thin lens focusing we can directly apply the result of the drifting beam. We find that, for a given transport length, fewer lenses and larger beam size oscillations will produce a better emittance at the end of the transport line provided that the beam makes an integer number of oscillations. Figures 11 and 12 show two simulations of a beam with the same initial conditions and transported through the same length of drift. In the first there is one thin lens applied when $\sigma / \sigma_{0}=8.5$. In the second, in order to approximate a beam which is more closely matched to a uniform focusing channel, a lens is applied each time the beam size doubles its initial value. It is clear from the graphs that, when the beam is allowed to expand enough to take advantage of the tuck under effect observed in the drifting beam above, much of the emittance growth can be reversed when the beam is focused back down. In the case where the beam size oscillations are kept smaller we see that the emittance oscillates around its peak value but never drops to as low a level as in the first case.

The striking performance of the scheme shown in Fig. 11 for minimizing the emittance at the envelope

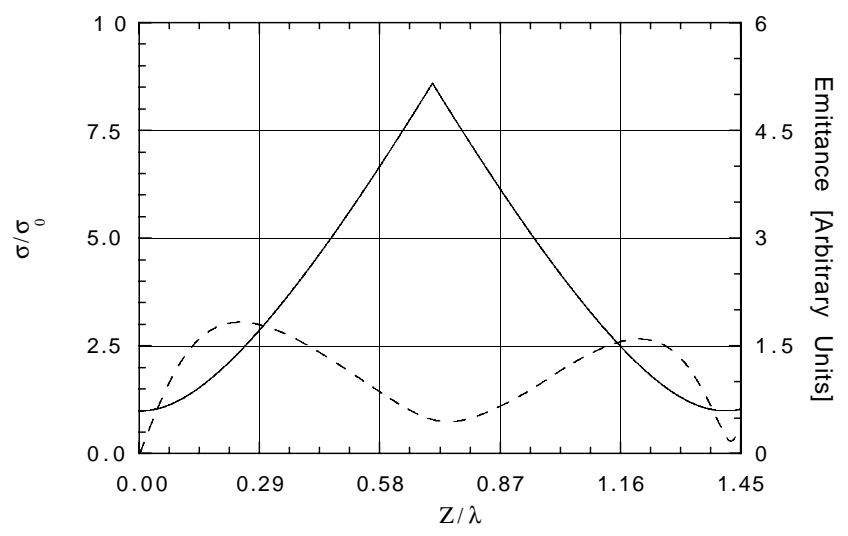

FIG. 11. Evolution of beam size and emittance in simulation with thin lens focusing applied at the point of initial emittance minimum. Lens strength chosen to reverse the envelope angle.

minimum - in other words, compensation of the nonlinear field-derived emittance-is understandable in a number of different ways. If the dynamics being described were only the linear slice dynamics, Figs. 2 and 3 illustrate that the emittance performance would be qualitatively the same in Figs. 11 and 12. They are not, however, and this is because of the strong wave breaking induced in the intraslice dynamics by the beam being too close to equilibrium. In other words, the existence of the offorigin moving "fixed point" in trace space gives rise to wave breaking, trace space filamentation, and associated irreversible emittance growth. O'Shea has identified irreversible emittance growth of this type with an increase in the entropy which, we note, is also equivalent to loss of order or information in the system. In the case of Fig. 11, the emittance increase due to field nonlinearities is reversed (compensated) and the information about the beam's initial state is preserved. An excellent illustration of this phenomenon is given in Fig. 13, which shows the beam distribution in $r$ at three points in the propagation

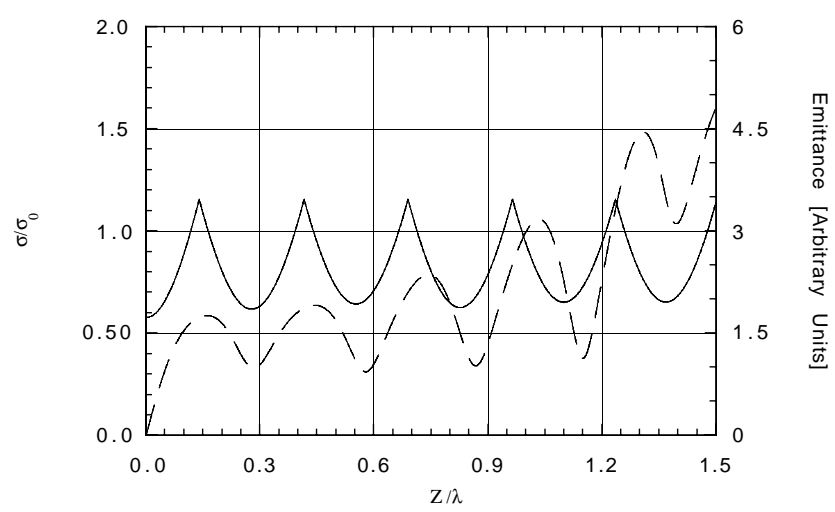

FIG. 12. Evolution of beam size and emittance in simulation with thin lens focusing applied at the points of beam envelope doubling and lens strength chosen to reverse the envelope angle. The simulation is followed for the same number of plasma periods as in Fig. 11. 

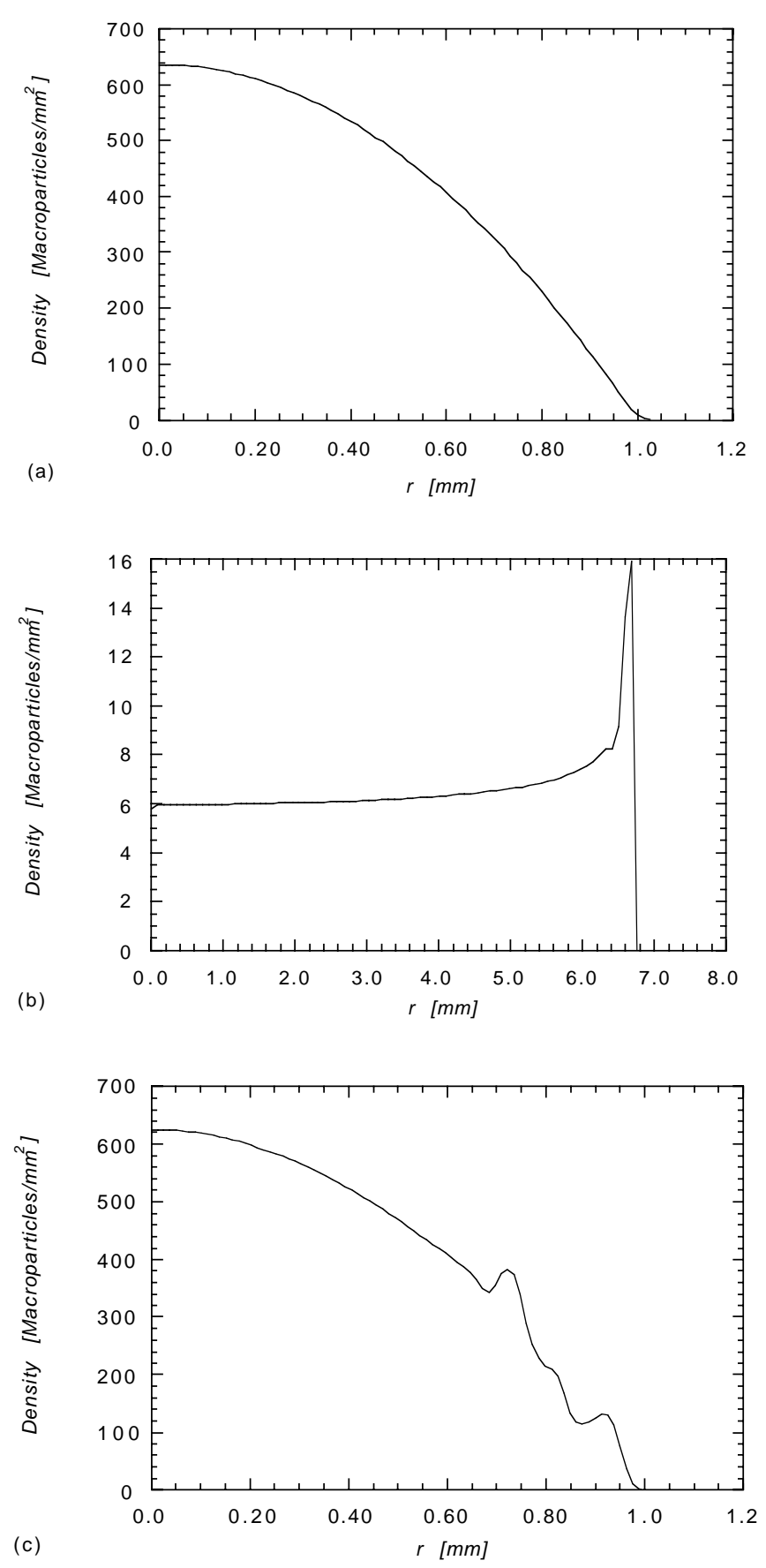

FIG. 13. Evolution of beam distribution during simulation shown in Fig. 11 at the (a) beginning, (b) focusing lens (midpoint), and (c) endpoint (emittance minimum).

of Fig. 11 -the initial and final states, as well as the thin lens position. It can be seen that, by this judicious choice of focusing, the final beam distribution reproduces the initial distribution remarkably well (information is retained during the beam oscillation), considering how distorted it becomes in intermediate points in the propagation.

It is useful at this point to make a connection between our terminology, based on collective space-charge forces

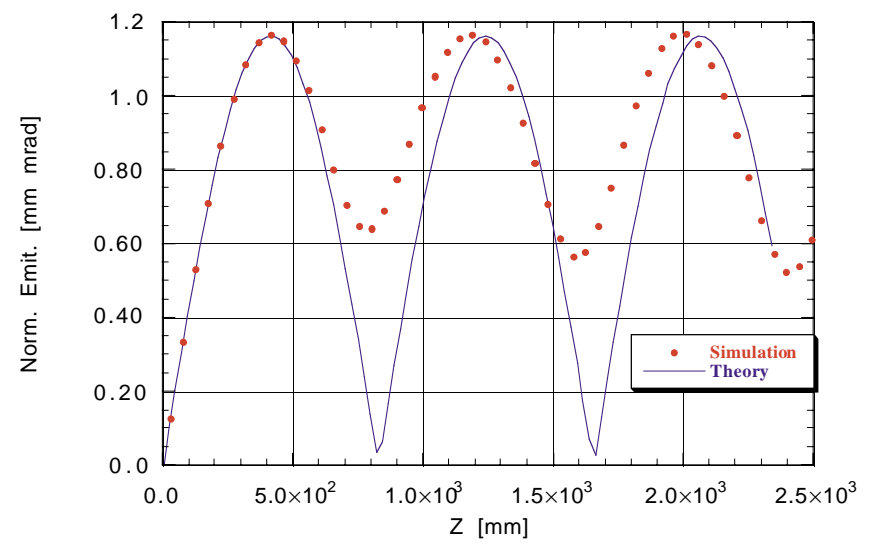

FIG. 14. (Color) Evolution of emittance for beam rms matched to a uniform focusing channel from simulation and analytical prediction [Eq. (50)].

and stated in terms of plasma frequencies, and the terminology of the ion-beam community, which emphasizes the optical properties of the periodic focusing system used. Periodic focusing systems are parameterized, in the limit of no collective forces (emittance dominated beam), by the betatron phase advance per period $\mu$ The case shown in Fig. 11, where the beam expands dramatically between lenses, is one in which $\mu$ is undefined, i.e., the transport is unstable with no space-charge defocusing present, while the space-charge depressed phase advance is near vanishing. This situation is known to be prone to envelope instability [10], however, as well as halo formation. For long periodic transport systems, these properties would be highly undesirable, but for the one or two oscillation systems typified by photoinjectors (where the spacecharge tune depression is removed by acceleration) these effects do not have time to assert themselves. On the other hand, in the case shown in Fig. 12 where a large amount of irreversible emittance growth is observed due to wavebreaking effects, the $\mu$ is small compared to unity, and the envelope motion is stable.

It is natural to consider the limit suggested by the simulation of Fig. 12, in which the beam size does not vary - the case of a beam matched in the rms sense to a uniform solenoidal focusing channel. We can also compare these simulations with the prediction of Eq. (50), at least until the onset of wave breaking. The emittance evolution found by simulation of an initially parabolic beam rms matched to a focusing channel along with the emittance predicted by Eq. (50) is shown in Fig. 14. Note that the emittance again follows the same pattern shown above in that it increases rapidly in a quarter of a plasma oscillation to a maximum [6]. Since wave breaking does not occur until this maximum is reached, the excellent agreement between theory and simulation up to that point is not surprising. We will encounter a similar type of emittance behavior in accelerating systems in the following sections. 


\section{LAMINAR AND NONLAMINAR MOTION IN ACCELERATING CYLINDRICAL BEAMS}

In the case of a beam accelerating under the influence of radio frequency fields, the paraxial equation of motion for a particle in a laminar flow condition now contains terms arising from adiabatic damping and ponderomotive (alternating transverse gradient) forces,

$$
r^{\prime \prime}(z)+\left(\frac{\gamma^{\prime}}{\gamma(z)}\right) r^{\prime}(z)+\frac{\eta}{8}\left(\frac{\gamma^{\prime}}{\gamma(z)}\right)^{2} r(z)=\frac{2 r_{e} \lambda\left(r_{0}\right)}{\gamma(z)^{3} r},
$$

which is again a nonlinear equation without an analytical solution. In this system, there is also an equilibriumlike particular solution to Eq. (51), which is analogous to the invariant envelope above, corresponding to each value of $r_{0}$.

As in previous sections, we proceed by finding an analytical formula for the emittance of a "matched" beam. In the case of an accelerating beam, we mean matched in the sense that the rms size of the beam follows the invariant envelope. This situation is slightly different from that of coasting beams because we are required to reference $\sigma^{\prime}$ to the nonstationary particular solution

$$
\begin{aligned}
r_{p}\left(r_{0}, z\right) & =\frac{4}{\gamma^{\prime}} \sqrt{\frac{r_{e} \lambda\left(r_{0}\right)}{(2+\eta) \gamma(z)}} \\
& \equiv r_{0} \frac{\bar{k}_{p}\left(r_{0}\right)}{k_{\beta}} \sqrt{\frac{1}{2+\eta} \frac{\gamma_{0}}{\gamma(z)}} .
\end{aligned}
$$

In Eq. (52) we have identified $k_{\beta}=\gamma^{\prime} / \sqrt{8} \gamma$, and can see that the particular solution is again proportional to the initial ratio $\bar{k}_{p}\left(r_{0}\right) / k_{\beta}$. We can again proceed to linearize Eq. (52) about these particular solutions to obtain

$$
\delta r^{\prime \prime}+\left(\frac{\gamma^{\prime}}{\gamma}\right) \delta r^{\prime}+\frac{1+\eta}{4}\left(\frac{\gamma^{\prime}}{\gamma}\right)^{2} \delta r=0,
$$

where $\delta r=r-r_{p}$. This equation has a general form of solution similar to that given by Eqs. (12) and (13). Therefore, we can solve Eq. (51) to find the single particle motion, yielding

$$
r\left(r_{0}, z\right)=r_{p}\left(r_{0}, z\right)+\left[r_{0}-r_{p 0}\left(r_{0}\right)\right] \cos \left[\frac{\sqrt{1+\eta}}{2} \ln \left(\frac{\gamma_{0}}{\gamma}\right)\right]+\frac{1}{\sqrt{1+\eta}}\left[r_{0}-r_{p 0}\left(r_{0}\right)\right] \sin \left[\frac{\sqrt{1+\eta}}{2} \ln \left(\frac{\gamma_{0}}{\gamma}\right)\right]
$$

where the integration constants are chosen so that

$$
r_{0}^{\prime}=\frac{1}{2} \frac{\gamma^{\prime}}{\gamma} r_{0}
$$

The wave-breaking condition is now given by

$$
\frac{\partial r_{p}}{\partial r_{0}}=-\frac{\cos \left[\frac{\sqrt{1+\eta}}{2} \ln \left(\frac{\gamma_{0}}{\gamma}\right)\right]}{2 \sin ^{2}\left[\frac{\sqrt{1+\eta}}{8} \ln \left(\frac{\gamma_{0}}{\gamma}\right)\right]} .
$$

The quantity on the right-hand side of Eq. (56) can be recast to give

$$
\begin{aligned}
\frac{\partial r_{p}}{\partial r_{0}} & =\frac{4 \pi r_{0} n_{b}}{\gamma} \sqrt{\frac{r_{c}}{(2+\eta) \lambda\left(r_{0}\right) \gamma(z)}} \\
& =\frac{k_{p}^{2}\left(r_{0}\right)}{2 k_{\beta} \gamma^{\prime}} \sqrt{\frac{\eta}{2+\eta}} \\
& =-\frac{\cos \left[\frac{\sqrt{1+\eta}}{2} \ln \left(\frac{\gamma_{0}}{\gamma}\right)\right]}{2 \sin ^{2}\left[\sqrt{\frac{1+\eta}{8}} \ln \left(\frac{\gamma_{0}}{\gamma}\right)\right]},
\end{aligned}
$$

and we see that wave breaking is again averted by cutting the tails off of the distribution.

To proceed in the analysis, we again use the laminarity condition to integrate over the initial beam distribution and determine the second moments of the distribution and the emittance. We find that the geometric emittance evolution for a beam rms matched to the invariant envelope is

$$
\varepsilon_{\text {geom }}=\frac{4 \alpha r_{e} \lambda_{b}}{\gamma^{\prime} \sqrt{\pi(1+\eta)} \gamma_{0} \gamma^{3}}\left|\sin \left[\frac{\sqrt{1+\eta}}{2} \ln \left(\frac{\gamma_{0}}{\gamma}\right)\right]\right|
$$

The subscript indicating that the emittance is the geometric measure is included here to differentiate this measure of trace space area from the normalized emittance $\left(\varepsilon_{m}=\beta \gamma \varepsilon_{\mathrm{geom}} \cong \gamma \varepsilon_{\mathrm{geom}}\right)$ which measures the beam's phase space area. In Eq. (58), as before, $\alpha$ is a unitless constant depending on the initial beam distribution, with values listed in Table III.

The expression for the emittance evolution given in Eq. (58) is valid (in the linear approximation $|\delta r| \ll r_{p}$ ) up to the wave-breaking point. The details of wave breaking in the accelerating beam system are discussed in the following section. Note that the emittance for this case is inversely dependent on the acceleration gradient $\gamma^{\prime}$ and proportional to the beam current. These dependences are due primarily to the setting of the beam size with the invariant envelope.

TABLE III. Values of the form factor $\alpha$ for various initial cylindrical beam slice distribution types, accelerating case.

\begin{tabular}{ll}
\hline \hline Distribution type & $\alpha$ \\
\hline Gaussian & 0.1704 \\
Parabolic & 0.0561 \\
Flattop & 0 \\
\hline \hline
\end{tabular}




\section{SIMULATION OF ACCELERATING CYLINDRICAL BEAMS}

In this section we study the behavior of an initially parabolic profile accelerating beam matched in the rms sense to the invariant envelope and compare simulation to analytical results. Figure 15 shows the simulation of normalized emittance evolution in such a case, along with emittance predicted by Eq. (58). Again we see that the normalized emittance rapidly increases to a local maximum. We also see from the figure that the analytical formula for the emittance agrees well with the simulation up to the emittance maximum. However, because Eq. (53) is the linearized equation of motion, and $|\delta r|$ has constant amplitude, while $r_{p} \propto \gamma^{-1 / 2} \propto z^{-1 / 2}$ decreases, the agreement between theory and simulation is not as striking as with the coasting beam. Also, we see that theory and simulation do not agree after the emittance maximum. This is in keeping with the coasting beam results, as the beam undergoes wave breaking near the emittance maximum and the assumption of laminarity used in Eqs. (51)-(58) is no longer true. This wave breaking is easily seen in the beam trace space at the peak emittance shown in Fig. 16.

We observe in Fig. 15 that the emittance does not change significantly shortly after the emittance maximum. Since the transverse plasma frequency of the beam decreases as $-\gamma^{3 / 2}$, the acceleration process essentially stops the plasma oscillations and the beam becomes emittance dominated. The initial emittance growth caused by spacecharge field nonuniformities then is "frozen in" and the

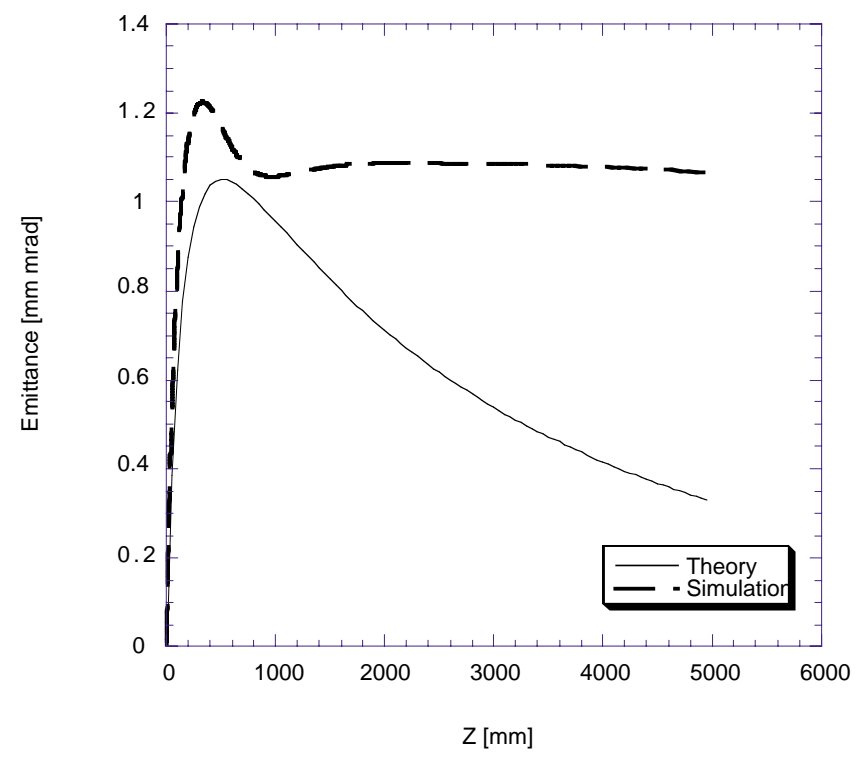

FIG. 15. (Color) Emittance evolution of an initially parabolic beam matched to the invariant envelope with a $60 \mathrm{MV} / \mathrm{m}$ peak accelerating field gradient. (These beam and accelerator parameters are the same as those in the booster linac at the Neptune Advanced Accelerator Laboratory [17].) The dashed line is the simulation result and the solid line is produced by Eq. (58).

beam has a finite irreversible emittance. We can use Eq. (58) to estimate the final emittance of the beam and, therefore, its size in the emittance dominated limit. To do this we start by finding the position of the emittance maximum,

$$
\begin{aligned}
\frac{\partial \varepsilon_{n}^{2}}{\partial z} & =0 \\
& =-\frac{16 \alpha^{2}}{(1+\eta) \pi \gamma_{0} \gamma^{\prime}}\left(\frac{r_{e} \lambda_{b}}{\gamma^{2}}\right)^{2} \sin \left[\frac{\sqrt{1+\eta}}{2} \ln \left(\frac{\gamma_{0}}{\gamma}\right)\right]\left\{\sin \left[\frac{\sqrt{1+\eta}}{2} \ln \left(\frac{\gamma_{0}}{\gamma}\right)\right]+\sqrt{1+\eta} \cos \left[\frac{\sqrt{1+\eta}}{2} \ln \left(\frac{\gamma_{0}}{\gamma}\right)\right]\right\},
\end{aligned}
$$

or

$$
\tan \left[\frac{\sqrt{1+\eta}}{2} \ln \left(\frac{\gamma_{0}}{\gamma}\right)\right]=-\sqrt{1+\eta} .
$$

Equation (60) yields the position of the emittance maximum,

$$
z_{\varepsilon_{\max }}=\frac{\gamma_{0}}{\gamma^{\prime}}\left[\frac{1}{e^{\left[2 \tan ^{-1}(-\sqrt{1+\eta})\right] / \sqrt{2}}}-1\right],
$$

and the maximum emittance at this point is simply

$\varepsilon_{n, \text { max }}=\frac{4 \alpha r_{e} \lambda_{b}}{\gamma^{\prime} \gamma_{0} \sqrt{\pi(1+\eta)}} \frac{\sin \left[\tan ^{-1}(-\sqrt{1+\eta})\right]}{\left\{\exp \left[\sqrt{2} \tan ^{-1}(\sqrt{1+\eta})\right]\right\}^{1 / 2}}$.

The final beam size in the simulations is estimated by ignoring the space-charge term in the envelope equation and assuming a steady state solution based on a constant normalized emittance equal to the maximum as predicted by Eq. (62),

$$
\sigma_{\min }=\left(\frac{8}{\eta}\right)^{1 / 4} \sqrt{\frac{\varepsilon_{n, \max }}{\gamma^{\prime}}} .
$$

A comparison between the final rms beam size achieved in simulation and the prediction of Eq. (63) for the simulation case of Fig. 16 is shown in Fig. 17. The agreement is remarkably good in the asymptotic region, where the simulated beam size approaches a constant value very close to that predicted from the analytical result of Eq. (63). Thus, one can determine the final beam characteristics simply by knowing the degree of nonuniformity of the initial distribution (which is parameterized by $\alpha$ ) at the beginning of acceleration with transverse matching to the invariant envelope. 


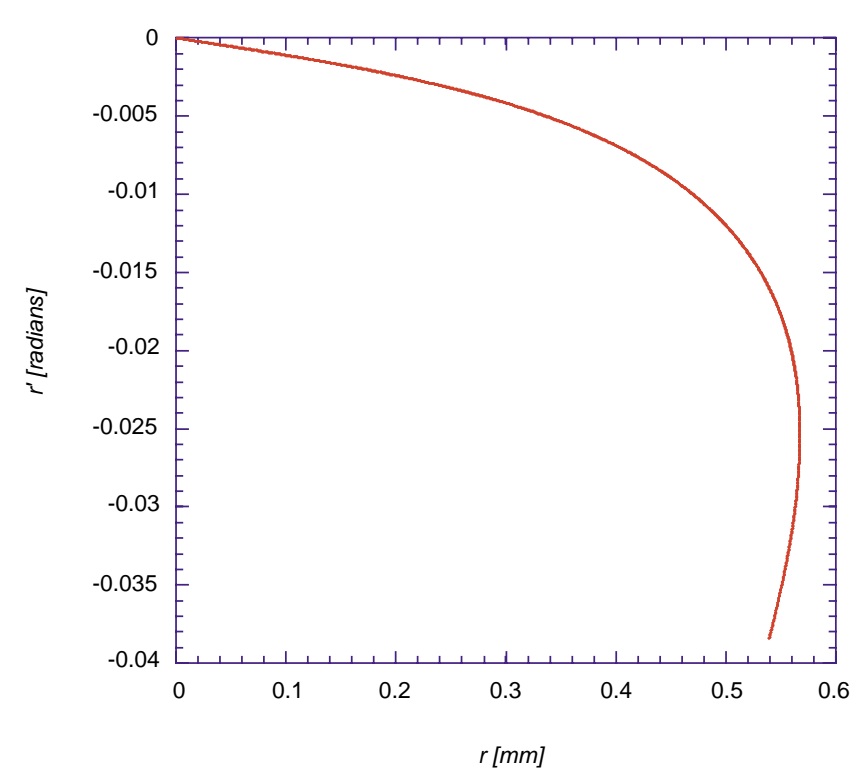

FIG. 16. (Color) Trace space of an initially parabolic beam slice at the maximum emittance point in the accelerating beam simulation. Wave breaking has just occurred.

As an example of the potential final emittance, we take the nominal linear coherent light source (LCLS) photoinjector design parameters [18], in which a $100 \mathrm{~A}$ beam is emitted in a high gradient rf gun, accelerated to $\gamma_{0} \approx 14$, and then focused into a matched invariant envelope at the beginning of a high gradient linac. For a standard SLAC $S$-band traveling wave $(\eta \cong 0.3)$ linac (average accelerating gradient of $17 \mathrm{MeV} / \mathrm{m}$ ), one obtains an asymptotic emittance of $\varepsilon_{n, \max }=6.5 \alpha \mathrm{mm}$ mrad. Even though a roughly uniform beam is planned to be launched at the cathode in this device, it will be nonuniform at the injection to the linac due to nonlinearities in the space-charge forces at very low velocities, as well as imperfections in the drive laser spatiotemporal profile. To see the potential effects of such nonlinearities, assumption of $\alpha \cong 0.1$

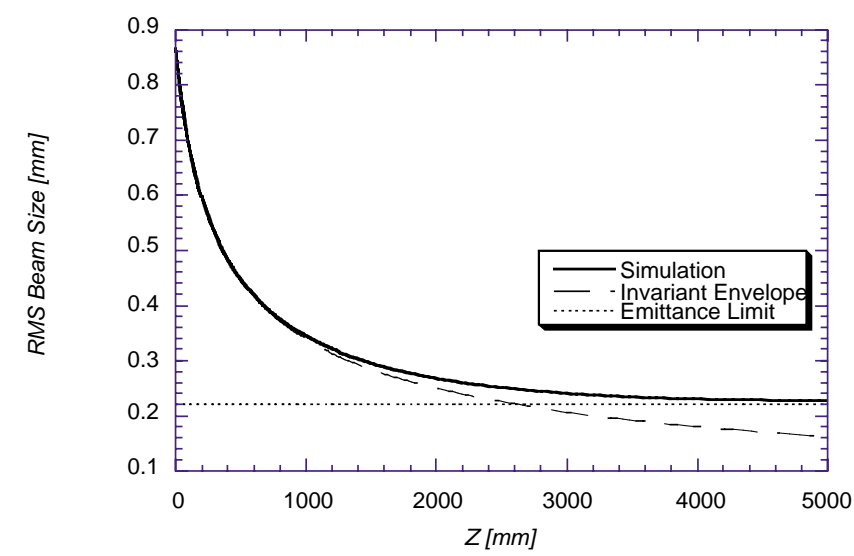

FIG. 17. (Color) The beam envelope evolution for the same simulation as Fig. 16. Here the beam size follows the invariant envelope initially, but levels off as it approaches the limit predicted by Eq. (63). (between a Gaussian and a parabolic profile) gives a predicted emittance due to nonlinearities alone of $\varepsilon_{n, \max }=0.65 \mathrm{~mm} \mathrm{mrad}$, which is nearly equal to the full allowed design emittance in the LCLS. An alternative design, which is discussed below, uses the high gradient (29 MeV/m average) standing wave $(\eta \cong 1)$ plane-wave transformer (PWT) linac developed at the UCLA Neptune Laboratory [17] for acceleration after the gun. In this case, we have $\varepsilon_{n, \max }=2.75 \alpha$, which produces a more tolerable margin for emittance due to nonlinearities and wave breaking.

\section{EMITTANCE COMPENSATED THREE-DIMENSIONAL BEAM SIMULATIONS}

The results of the one-dimensional beam distribution (with $z$ dependent dynamics) analyses discussed above were introduced, of course, to aid in explaining the physics of beams with truly three-dimensional distributions, such as are found in short-pulse photoinjectors. An interesting photoinjector design has just been developed in context of the LCLS x-ray free-electron laser (FEL) injector collaboration [18], in which an ultrahigh gradient rf gun is followed by two short $(42 \mathrm{~cm})$ PWT sections to bring the beam to $33 \mathrm{MeV}$ final energy. This design was originally found by use of a linear "slice" simulation code, termed HOMDYN [19]. In the case of the LCLS photoinjector, the design parameters predicted by HOMDYN were verified by multiparticle simulation, as we shall see below, to be excellent choices. The design assumes a perfectly uniform beam emitted from the cathode (constant current density up to hard boundaries in radius and time), and thus is modeled well by HOMDYN, which assumes the same scenario. On the other hand, we are presently concerned with beam densities with nontrivial radial dependences, which we have found to give rise to both reversible and irreversible emittance growth. Thus, in order to illuminate the role of the nonlinear intraslice forces we have examined here, we compare cases with radially uniform and nonuniform emission via three-dimensional, self-consistent multiparticle PARMELA simulation.

We begin by illustrating the dynamics of the rms beam size and normalized rms emittance in the case of a perfectly uniform beam injected at the photocathode in Fig. 18. The pulse is uniform over $10 \mathrm{psec}$ and a radius of $1 \mathrm{~mm}$, and vanishes outside of these boundaries. The total charge injected is $1 \mathrm{nC}$, and the peak accelerating fields in the rf gun and PWTs are 140 and $58 \mathrm{MV} / \mathrm{m}$, respectively. The focusing solenoid is adjusted to produce a matched invariant envelope in the PWT post-acceleration sections. Figure 18 shows the extremely good rms normalized emittance achieved $(0.4 \mathrm{~mm}$ mrad) in this design after a final drift to roughly $4 \mathrm{~m}$ from the cathode. It should be noted that, as one might expect from the simulations shown in Fig. 11, the size of the beam on the cathode and its size at the first compensation point (end of first beam size 


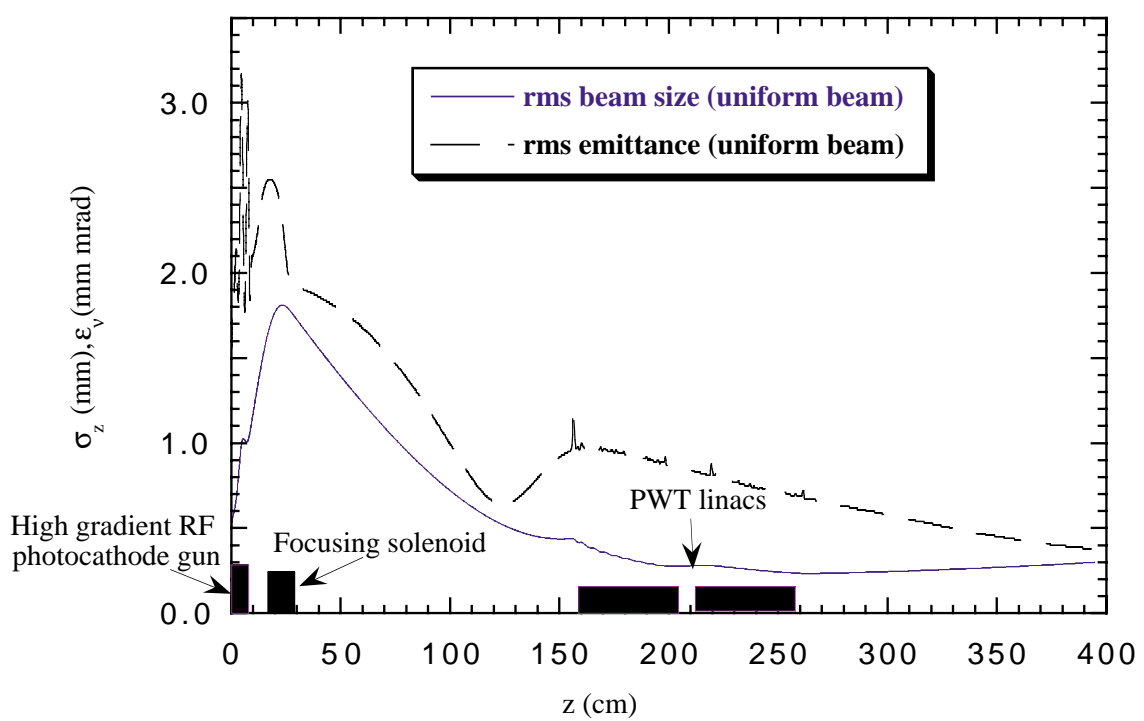

FIG. 18. (Color) Results of a PARMELA simulation of a uniform beam emitted at the cathode in the LCLS photoinjector design, with a 1.6 cell rf gun followed by an emittance-compensating solenoid and two PWT standing wave sections.

oscillation) are nearly identical in this optimized design. This fact points to the close relationship that interslice (linear) and intraslice (nonlinear) emittance oscillations have with each other-they are both governed by the same oscillation frequency but, in contrast to the intraslice emittance growth, the interslice dynamics are always reversible.

The $(x, z)$ spatial profile of the 10000 simulation particles at the $z=4 \mathrm{~m}$ point is shown in Fig. 19. It can be seen that, while the core of the beam is well behaved in terms of the different $z$ slices ending up in the same configuration, the leading and trailing beam edges display very different behavior. This is due to the fact that the transverse space-charge forces drop dramatically in these longitudinal "tail" regions, and the particles in these slices do not focus to space-charge dominated waists, but actually cross the beam axis. This is clearly an example of nonlaminar flow, and, as a result, the total transverse phase space bifur-

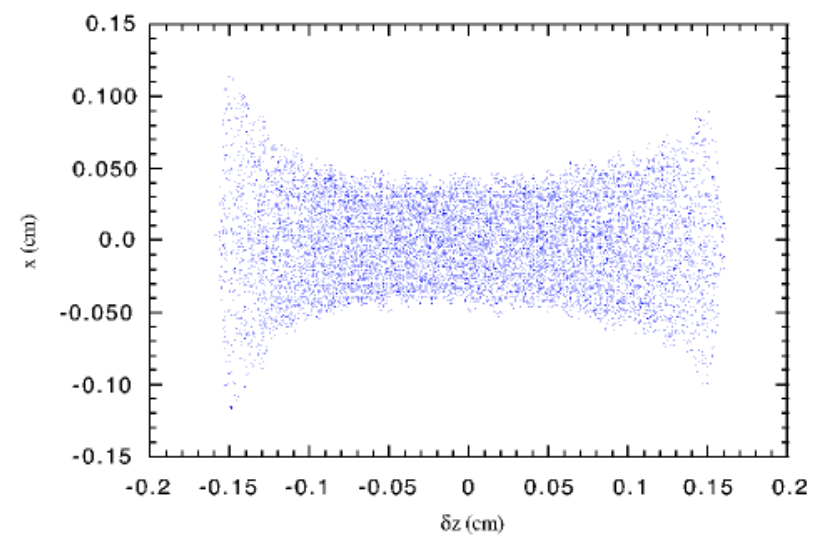

FIG. 19. (Color) Spatial $(x, z)$ distribution at the end of the PARMELA simulation shown in Fig. 18. cates into two populations. One population is composed of slices whose dynamics were described in Sec. II, and has essentially no wave breaking associated with it, and another in which beam particles wave break near the trace space origin (not off axis, as our analysis in preceding sections has examined).

These populations can also be observed in the $(x, y)$ spatial distribution shown in Fig. 20(a) and the phase space distribution displayed in Fig. 21(a). In Fig. 20(a) the bifurcated population produces a slight beam halo, while in Fig. 21(a) one can directly see the bifurcation as distinct lengths of the slices in phase space. These points are emphasized by Figs. 20(b) and 21(b), in which the $(x, y)$ spatial and phase space distributions are shown for the beam population located only within $\delta z= \pm 0.1 \mathrm{~mm}$ from the beam longitudinal center. In Fig. 20(b), the beam halo essentially disappears when this cut is made. In Fig. 21(b), one sees a very interesting situation-even though the longitudinal tails have gone bifurcated, they are realigned in phase space with the beam cores. The difference between the bifurcated and unbifurcated populations is simply that the length in phase space is larger for the bifurcated population in the longitudinal tails.

This dynamical picture changes significantly if one injects a beam with a radially nonuniform current profile (but same rms beam dimensions), as is the case in the simulation results shown in Fig. 22. Here a radial Gaussian profile, cutoff at $r=\sigma$, is introduced at the cathode. While the rms beam envelope does not change appreciably with a nonuniform injected beam, as is well known in the theory of space-charge dominated beams [10], the phase space dynamics reveal many changes from the uniform beam case, as can be seen by examining the rms emittance evolution in Fig. 22. As expected, the emittance grows significantly when nonlinearities in the space-charge field 

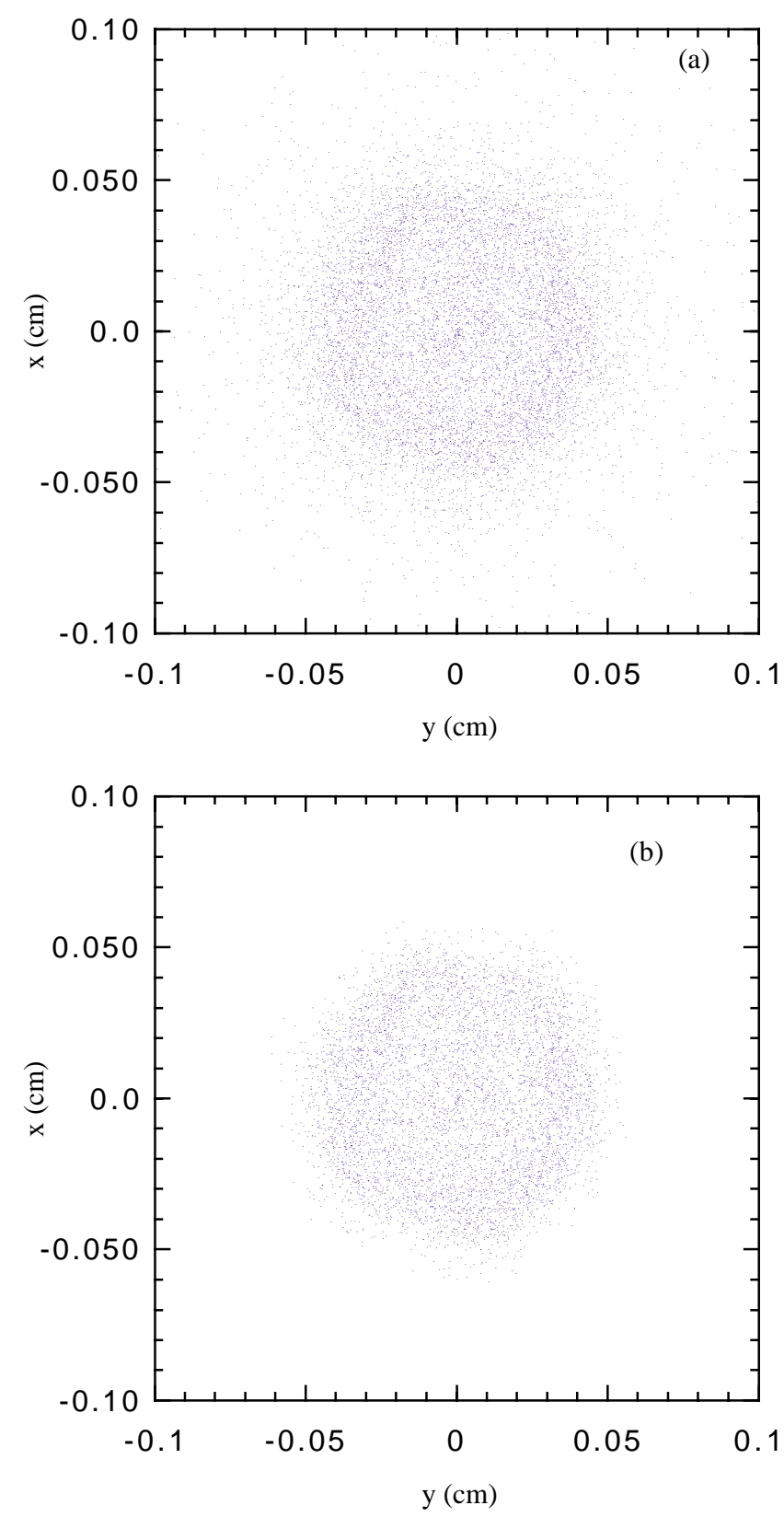

FIG. 20. (Color) (a) Spatial $(x, y)$ distribution at the end of the PARMELA simulation shown in Fig. 18. (b) Spatial $(x, y)$ distribution at the end of the PARMELA simulation shown in Fig. 18, with a cut made on the distribution at $\delta z= \pm 0.1 \mathrm{~mm}$ to remove longitudinal tails.

are enhanced by the nonuniformity of the beam's radial distribution. Also, as expected, the total emittance of the beam in this case is dominated by the slice emittance and not by the differential phase space angles of each slice. The degree to which this is the case can be deduced by examination of the spatial $(x, z)$ distribution at the end of the simulation shown in Fig. 23. In this configuration space picture, we see that the strong difference in transverse profile as a function of $z$ is greatly mitigated when the beam is no longer radially uniform at injection.
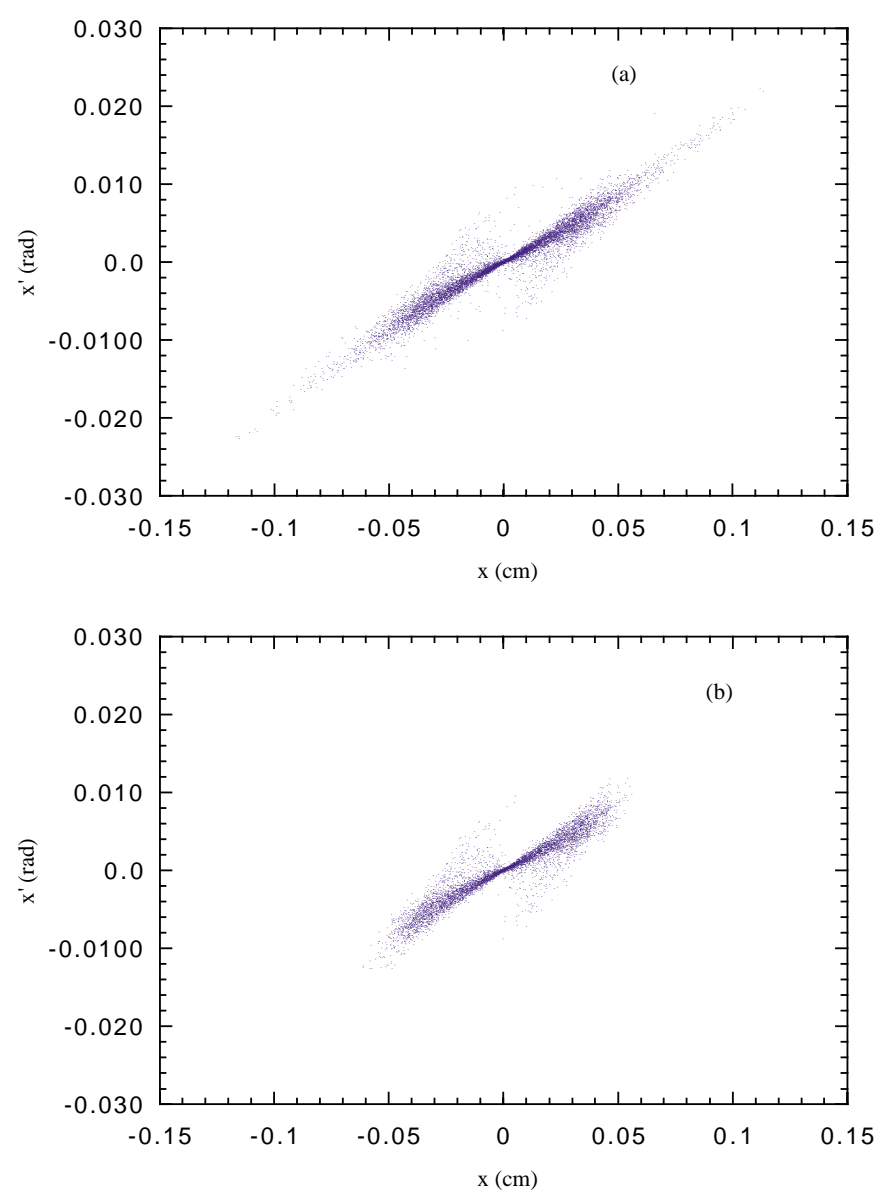

FIG. 21. (Color) (a) Phase space distribution at the end of the PARMELA simulation shown in Fig. 18. (b) Phase space distribution at the end of the PARMELA simulation shown in Fig. 18, with a cut made on the distribution at $\delta z= \pm 0.1 \mathrm{~mm}$ to remove longitudinal tails.

Figure 23 also displays the existence of a long transverse halo. This halo, along with the general nonuniformity of the $(x, y)$ spatial distribution at the end of the simulations, is also shown well in Fig. 24(a). The halo is quite uniformly contributed from all $z$ slices of the beam, as can be deduced as well from Fig. 24(b), where the $(x, y)$ spatial distribution is replotted after making cuts at $\delta z= \pm 0.1 \mathrm{~mm}$. It can be seen from comparison of Figs. 24(a) and 24(b) that the transverse halo does not preferentially arise from the longitudinal tails of the beam.

This conclusion is strongly reinforced by the phase space plots shown in Fig. 25(a) (full beam) and 25(b) (distribution cut at $\delta z= \pm 0.1 \mathrm{~mm}$ ). The full and cut phase spaces are virtually identical, as are the normalized $\mathrm{rms}$ emittances $\varepsilon_{n}=2 \mathrm{~mm}$ rad for the full beam, $1.8 \mathrm{~mm}$ mrad for the cut distribution). The lack of notably different behavior between the longitudinal beam core and tails in the nonuniform beam is a result of the mitigation of an unphysical discontinuity in the beam profile. Thus we expect the results of our one-dimensional 


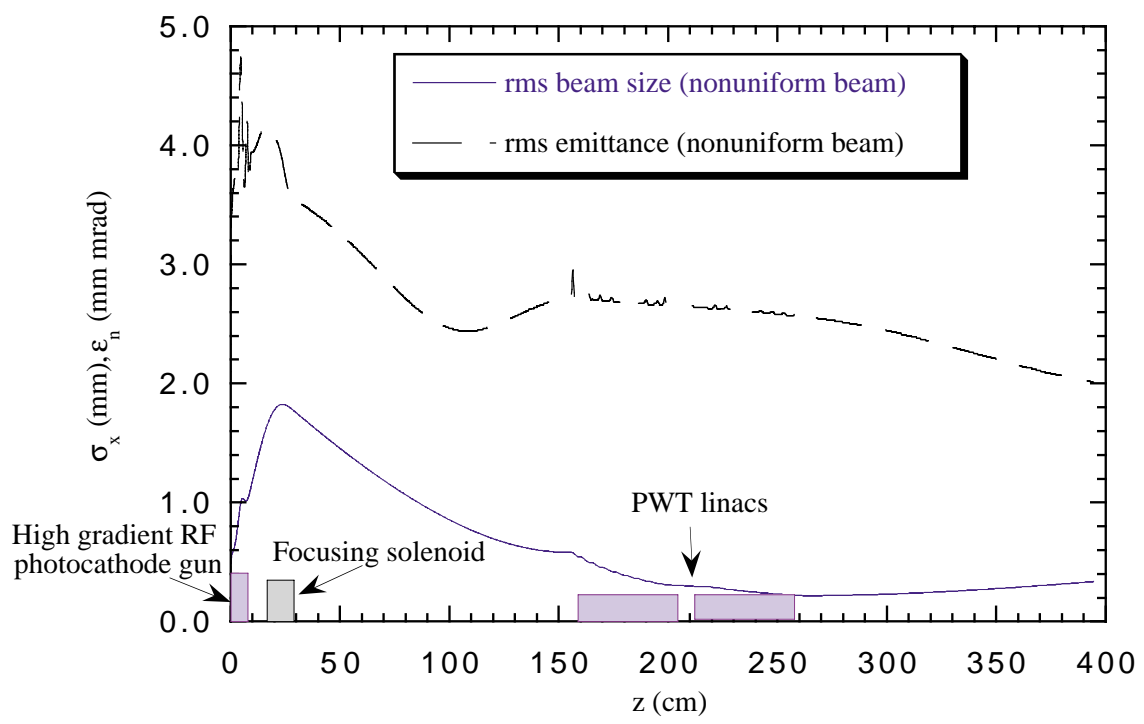

FIG. 22. (Color) Results of a PARMELA simulation of a radially nonuniform beam (Gaussian, cutoff at $r=\sigma$ ) emitted at the cathode for the same accelerator conditions as in Fig. 18.

(purely radial) dynamics analysis to be surprisingly more applicable to modeling of finite length photoinjector beams than might be deduced from the purely uniform beam case.

The phase space pictures shown in Fig. 25 display again a bifurcation of particle populations, along with the long halo of particles at large amplitude in phase space. This bifurcation is essentially not due to differential slice motion in this case, but is due to wave breaking. The components of the beam which have not undergone wave breaking are localized within $\left|x^{\prime}\right|<10 \mathrm{mrad}$, while the components which have undergone wave breaking display large angles and offsets in $x$.

The phase space distributions shown in Fig. 25 are complicated pictures, which result from two separate folding bifurcations of the transverse distribution within a slice.

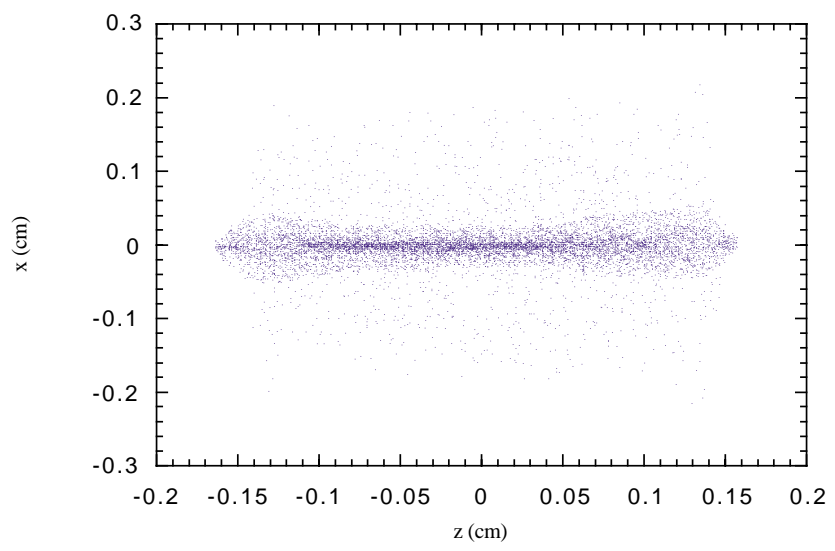

FIG. 23. (Color) Spatial $(x, z)$ distribution at the end of the PARMELA simulation shown in Fig. 22
The core of the beam, which is inside of the initial wavebreaking point, is well behaved, laminar, and aligned to near the $x$ axis. On the other hand, the particles which begin outside of the initial wave-breaking point fold twice: first these particles "tuck" under the core of the distribution, and then the ones at largest initial $x$ move quickly across the $x=0$ axis to large amplitude and positive $\left(x, x^{\prime}\right)$ correlation. The particles at intermediate initial values of $x$ between these two cases are almost, but not quite, prevented by the space-charge forces from crossing the $x=0$ axis, and end up nearly aligned to this $\left(x^{\prime}\right)$ axis. Thus the characteristic "cross" shape within a large amplitude halo seen in Fig. 25 is formed. This elaborate phase space picture of course implies that a large amount of irreversible emittance growth has occurred. The differences between Figs. 21 and 25 illustrate well one of the main points of this paper - the onset of wave breaking and emittance growth which results from matching of a beam to a generalized transverse equilibrium.

We also note that the emittance as shown in Fig. 22 is much larger than the sum of the emittance in the uniform beam case with the estimate of Eq. (62) added to it. The extra component of the emittance given in Eq. (62) is due only to wave breaking occurring after entrance into the first PWT linac and ignores the emittance one may expect from the initial beam oscillation from the cathode to this point. We have not provided an emittance estimate from the nonlinear intraslice dynamics during this oscillation. This is in part because our previous analyses do not allow a good modeling of cylindrically symmetric beams so far from equilibrium, and also because the transverse fields of the beam very near to the cathode are not of simple quasione-dimensional form, as is also assumed in our previous analyses. 

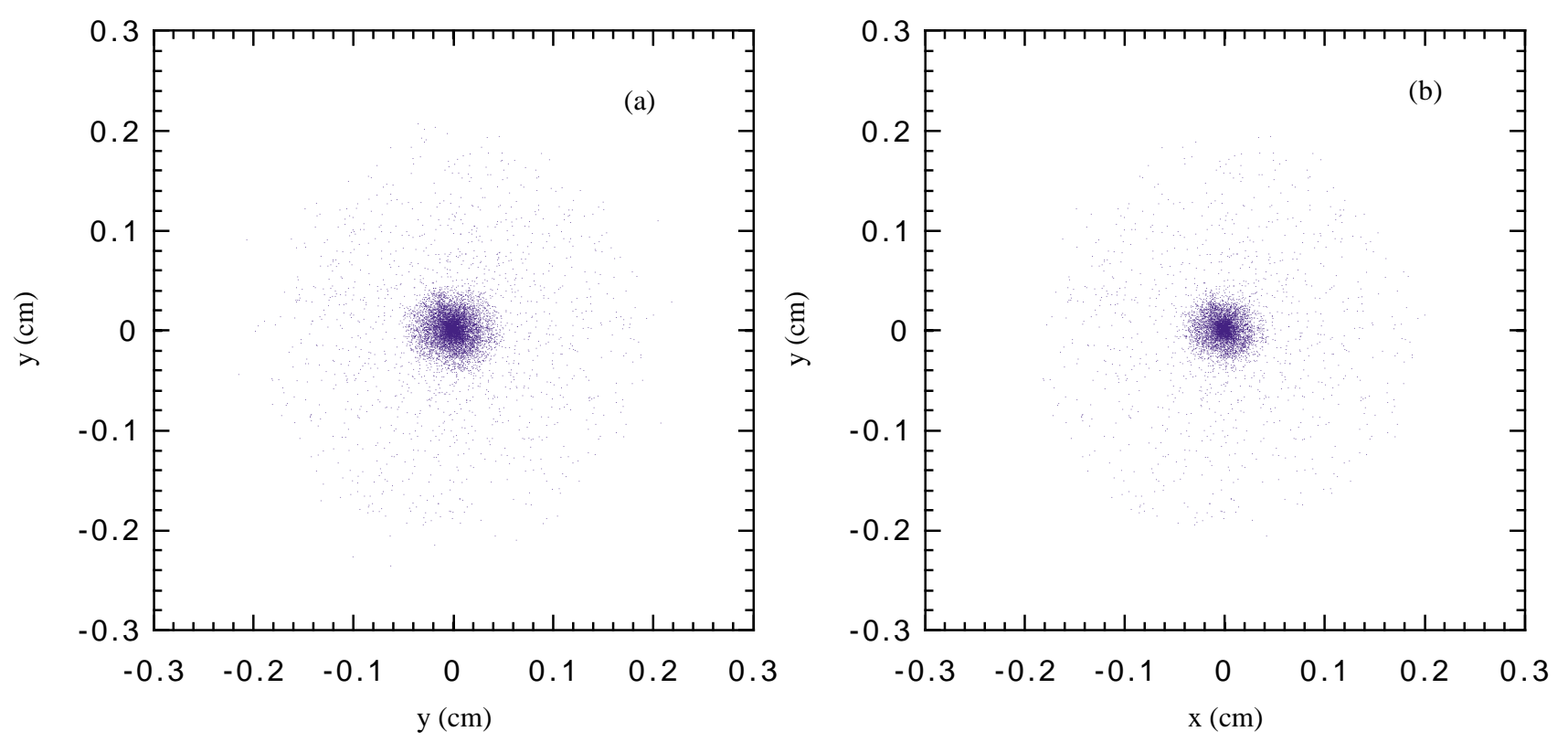

FIG. 24. (Color) (a) Spatial $(x, y)$ distribution at the end of the PARMELA simulation shown in Fig. 22. (b) Spatial ( $x, y)$ distribution at the end of the PARMELA simulation shown in Fig. 22, with a cut made on the distribution at $\delta z= \pm 0.1 \mathrm{~cm}$ to remove longitudinal tails.
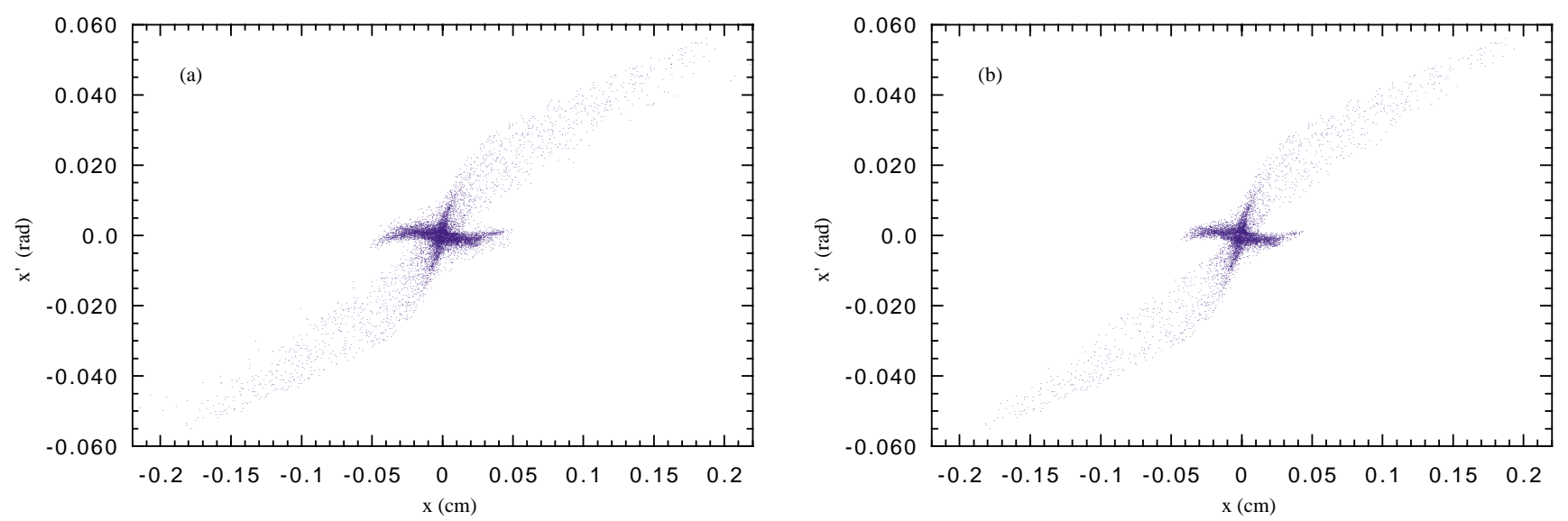

FIG. 25. (Color) (a) Phase space distribution at the end of the PARMELA simulation shown in Fig. 22. (b) Phase space distribution at the end of the PARMELA simulation shown in Fig. 22, with a cut made on the distribution at $\delta z= \pm 0.1 \mathrm{~cm}$ to remove longitudinal tails.

\section{CONCLUSIONS}

In this paper, we have explored the consequenceswave breaking and associate emittance growth-of the choice of beam envelope trajectory, i.e., the degree to which a beam is matched to a generalized equilibrium. In cases where the nonlinearity of the field is tolerable (as in the perfectly uniform beam simulated in Sec. VIII), running the beam essentially on the invariant envelope in a booster linac works well, as predicted by the analysis of SR. In the example of such a case (Figs. 18-21), it can be seen that very few nonuniformities are introduced into the distribution by the initial emittance oscillation, in which the beam leaves the cathode, experiences some non- negligible nonlinearities in the field for a short time, and is then accelerated and focused (to the same radius as at the cathode) to produce a beam which is appropriate for injection into the linac. This example illustrates one of the conclusions of our work-emittance compensation in cylindrically symmetric systems works well when the oscillation has a large amplitude, far from a matched condition. This conclusion is applicable to transport in a relatively low energy beam line (e.g., $20 \mathrm{MeV}$ in the Tesla Test Facility [20]) placed after an emittance compensated photoinjector.

On the other hand, when a moderately nonuniform beam is injected at the cathode, the initial emittance compensation is degraded and the "second compensation" 
achieved by matching to the invariant envelope is almost eliminated-in the uniform beam, the emittance diminishes by $60 \%$ in the second compensation, and in the nonuniform beam case it is diminished by only $20 \%$. The minimum emittance associated with the process illustrated by this example is given by Eq. (62), which serves a useful guide to estimation of the best performance possible for a given injector configuration.

In conclusion, in this work we have attempted to unify the microscopic concepts of linear emittance compensation, which arise in high brightness electron beam physics, and nonlinear wave breaking, which has had an impact on the understanding of ion beam physics, showing their relationship to one another in the context of high brightness photoinjectors. We have provided both a qualitative picture of extremely space-charge dominated beam dynamics in radially nonuniform beams and quantitative predictions concerning the irreversible emittance expected to arise due to wave breaking and subsequent phase space filamentation. This understanding aids in the classification of global characteristics of beam distributions, such as nonlinear field energy and entropy, which have been originally introduced in the field of intense ion beams. While it is clear that this work has imported some valuable concepts from this field into the study of the high brightness electron beams, it is not yet clear that our results are of other than conceptual significance to the study of intense ion beam transport. This question will be left to further investigations.

\section{ACKNOWLEDGMENT}

The authors wish to thank Luca Serafini for his guidance in the computational aspects of this work as well his interest and comments.
[1] K. J. Kim, Nucl. Instrum. Methods Phys. Res., Sect. A 275, 201 (1989).

[2] B. E. Carlsten, Nucl. Instrum. Methods Phys. Res., Sect. A 285, 313 (1989).

[3] X. Qiu et al., Phys. Rev. Lett. 76, 3723 (1996).

[4] Luca Serafini and J. B. Rosenzweig, Phys. Rev. E 55, 7565 (1997).

[5] L. Brillouin, Phys. Rev. 67, 260 (1945).

[6] O. A. Anderson, Part. Accel. 21, 197 (1987).

[7] T. P. Wangler et al., IEEE Trans. Nucl. Sci. 32, 2196 (1985).

[8] I. Hoffman and J. Struckmeier, Part. Accel. 21, 69 (1987).

[9] Patrick G. O'Shea, Phys. Rev. E 57, 1081 (1998).

[10] Martin Reiser, Theory and Design of Charged Particle Beams (Wiley, New York, 1994).

[11] T. P. Wangler, RF Linear Accelerators (Wiley, New York, 1998).

[12] S. C. Hartman and J. B. Rosenzweig, Phys. Rev. E 47, 2031 (1993).

[13] J. B. Rosenzweig and L. Serafini, Phys. Rev. E 49, 1599 (1994).

[14] P. Lapostolle, IEEE Trans. Nucl. Sci. 18, 1101 (1971).

[15] Bruce E. Carlsten, Phys. Rev. E 60, 2280 (1999).

[16] L. Serafini (private communication).

[17] J. B. Rosenzweig et al., Nucl. Instrum. Methods Phys. Res., Sect. A 410, 532 (1998).

[18] J. Arthur et al., SLAC Report No. SLAC-R-0521, 1998.

[19] M. Ferrario et al., in Proceedings of the ICFA Advanced Accelerator Workshop on the Physics of High Brightness Beams, Los Angeles, 1999 (World Scientific, Singapore, 2000).

[20] E. Colby et al., in Proceedings of the 1997 Particle Accelerator Conference, Vancouver, BC (IEEE, Piscataway, NJ, 1998). 